TRANSACTIONS OF THE

AMERICAN MATHEMATICAL SOCIETY

Volume 361, Number 3, March 2009, Pages 1597-1623

S 0002-9947(08)04533-9

Article electronically published on October 22, 2008

\title{
THE DYNAMICS OF MAPS TANGENT TO THE IDENTITY AND WITH NONVANISHING INDEX
}

\author{
LAURA MOLINO
}

\begin{abstract}
Let $f$ be a germ of a holomorphic self-map of $\mathbb{C}^{2}$ at the origin $O$ tangent to the identity, and with $O$ as a nondicritical isolated fixed point. A parabolic curve for $f$ is a holomorphic $f$-invariant curve, with $O$ on the boundary, attracted by $O$ under the action of $f$. It has been shown by M. Abate (2001) that if the characteristic direction $[v] \in \mathbb{P}\left(T_{O} \mathbb{C}^{2}\right)$ has residual index not belonging to $\mathbb{Q}^{+}$, then there exist parabolic curves for $f$ tangent to $[v]$. In this paper we prove, using a different method, that the conclusion still holds just assuming that the residual index is not vanishing (at least when $f$ is regular along $[v]$ ).
\end{abstract}

\section{INTRODUCTION}

One of the most interesting areas in the study of local dynamics in several complex variables is the dynamics near the origin $O$ of maps tangent to the identity; that is, of germs of holomorphic self-maps $f: \mathbb{C}^{n} \rightarrow \mathbb{C}^{n}$ such that $f(O)=O$ and $d f_{O}=$ id. When $n=1$ the dynamics is described by the celebrated Leau-Fatou flower theorem; when $n>1$ we are still far from understanding the complete picture, even though very important results have been obtained in recent years (see, e.g., 7], [14, 9], 2]).

In this paper we want to investigate conditions ensuring the existence of parabolic curves (the 2-variables analogue of the petals in the Leau-Fatou flower theorem) for maps tangent to the identity in dimension 2. Let us first recall some definitions and useful results concerning maps tangent to the identity. Let $f$ be a germ of a holomorphic self-map of $\mathbb{C}^{2}$ fixing the origin and tangent to the identity. We can write $f=\left(f_{1}, f_{2}\right)$, and let $f_{j}=z_{j}+P_{j, \nu_{j}}+P_{j, \nu_{j}+1}+\cdots$ be the homogeneous expansion of $f_{j}$ in a series of homogeneous polynomials, where $\operatorname{deg} P_{j, k}=k$ (or $\left.P_{j, k} \equiv 0\right)$ and $P_{j, \nu_{j}} \not \equiv 0$. The $\operatorname{order} \nu(f)$ of $f$ is defined by $\nu(f)=\min \left\{\nu_{1}, \nu_{2}\right\}$. We say that the origin is dicritical if we have $z_{2} P_{1, \nu(f)}\left(z_{1}, z_{2}\right) \equiv z_{1} P_{2, \nu(f)}\left(z_{1}, z_{2}\right)$.

A parabolic curve for $f$ at the origin is an injective holomorphic map $\varphi: \Delta \rightarrow \mathbb{C}^{2}$ satisfying the following properties:

(i) $\Delta$ is a simply connected domain in $\mathbb{C}$, with $0 \in \partial \Delta$;

(ii) $\varphi$ is continuous at the origin, and $\varphi(0)=O$;

(iii) $\varphi(\Delta)$ is invariant under $f$, and $\left(\left.f\right|_{\varphi(\Delta)}\right)^{n} \rightarrow O$ as $n \rightarrow \infty$.

Received by the editors April 8, 2005 and, in revised form, March 15, 2007. 2000 Mathematics Subject Classification. Primary 32H50, 37F10. 
Furthermore, if $[\varphi(\zeta)] \rightarrow[v] \in \mathbb{P}^{1}$ as $\zeta \rightarrow 0$, where $[\cdot]$ denotes the canonical projection of $\mathbb{C}^{2} \backslash\{O\}$ onto $\mathbb{P}^{1}$, we say that $\varphi$ is tangent to $[v]$ at the origin.

A characteristic direction for $f$ is a point $[v]=\left[v_{1}: v_{2}\right] \in \mathbb{P}^{1}$ such that there is $\lambda \in$ $\mathbb{C}$ so that $P_{j, \nu(f)}\left(v_{1}, v_{2}\right)=\lambda v_{j}$ for $j=1,2$. If $\lambda \neq 0$, we say that $[v]$ is nondegenerate; otherwise, it is degenerate. Characteristic directions arise naturally if we want to investigate the existence of parabolic curves tangent to some direction $[v]$. In fact, Hakim observed that if there exist parabolic curves tangent to a direction $[v]$, then this direction is necessarily characteristic (9]). However, Hakim was able to prove the converse for nondegenerate characteristic directions only:

Theorem 1.1 (Écalle, [7; Hakim, [9], 10]). Let $f$ be a (germ of a) holomorphic self-map of $\mathbb{C}^{2}$ fixing the origin and tangent to the identity. Then for every nondegenerate characteristic direction $[v]$ of $f$ there are $\nu(f)-1$ parabolic curves tangent to $[v]$ at the origin.

When $f$ has no nondegenerate characteristic directions, this theorem gives no information about the dynamics of $f$. Furthermore, there are examples of parabolic curves tangent to degenerate characteristic directions.

Example 1.2. Let us consider the germ $f$ given by

$$
\left\{\begin{array}{l}
f_{1}(z, w)=z+z w+w^{2}-z^{3}+O\left(z^{2} w, z w^{2}, w^{3}, z^{4}\right) \\
f_{2}(z, w)=w\left[1+z+w+O\left(z^{2}, z w, w^{2}\right)\right] .
\end{array}\right.
$$

We observe that $[1: 0]$ is a degenerate characteristic direction. The line $\{w=0\}$ is $f$-invariant, and inside it $f$ acts as the function $z-z^{3}+O\left(z^{4}\right)$. The classical Leau-Fatou theory then shows that there exist 2 parabolic curves for $f$ tangent to $[1: 0]$ at the origin $([8,[11])$.

A further step toward the understanding of the dynamics in a neighbourhood of an isolated fixed point has been done by Abate, who gave a complete generalization of the Leau-Fatou flower theorem in $\mathbb{C}^{2}$ :

Theorem 1.3 (Abate, [2]). Let $f$ be a (germ of a) holomorphic self-map of $\mathbb{C}^{2}$ tangent to the identity and such that the origin is an isolated fixed point. Then there exist (at least) $\nu(f)-1$ parabolic curves for $f$ at the origin.

The proof of this theorem is based on the possibility of modifying the geometry of the ambient space via a finite number of blow-ups, and of defining a residual index $\operatorname{lnd}(\tilde{f}, S, p) \in \mathbb{C}$, where $\tilde{f}$ is a holomorphic self-map of a complex 2-manifold $M$ which is the identity on a 1-dimensional submanifold $S$, and $p \in S$. It turns out that this index is either not defined anywhere on $S$, in which case we say that $\tilde{f}$ is degenerate along $S$ (or nontangential to $S$ in the terminology of [3], where it is described as a far-reaching approach to indices for holomorphic self-maps), or it is everywhere defined, and then we say that $\tilde{f}$ is nondegenerate along $S$ (respectively, tangential to $S$ ).

In particular, Abate gave a generalization of Theorem 1.1 to those characteristic directions whose residual index is not a nonnegative rational number.

Theorem 1.4 (Abate, 2]). Let $f$ be a (germ of a) holomorphic self-map of $\mathbb{C}^{2}$ tangent to the identity and such that the origin is an isolated fixed point. Let $[v]$ be a characteristic direction of $f$ such that $\operatorname{Ind}\left(\tilde{f}, \mathbb{P}^{1},[v]\right) \notin \mathbb{Q}^{+}$(here $\mathbb{P}^{1}$ is the 
exceptional divisor of the blow-up of the origin, and $\tilde{f}$ is the blow-up of $f$ ). Then there are (at least) $\nu(f)-1$ parabolic curves for $f$ tangent to $[v]$ at the origin.

The theory about the existence of parabolic curves tangent to a direction $[v]$, for maps tangent to the identity, is thus almost complete, but there are still examples where the previous results cannot be applied.

Example 1.5. Let us consider the map $f$ given by

$$
\left\{\begin{array}{l}
f_{1}(z, w)=z+z w+O\left(w^{2}, z^{3}, z^{2} w\right) \\
f_{2}(z, w)=w+2 w^{2}+b z^{3}+z^{4}+O\left(z^{5}, z^{2} w, z w^{2}, w^{3}\right),
\end{array}\right.
$$

with $b \neq 0$. We observe that $[v]=[1: 0]$ is a degenerate characteristic direction for $f$ with $\operatorname{Ind}\left(\tilde{f}, \mathbb{P}^{1},[v]\right)=1$. Hence, we cannot say anything about the dynamics of $f$ in the direction [1:0] using Theorem 1.1] or Theorem 1.4 .

As a corollary of our work (see Corollary 1.7) we shall be able to prove the existence of parabolic curves tangent to $[1: 0]$ also for this example (and many others). This will be a consequence of a more general result; to state it precisely we need to recall another set of definitions.

Any germ $g$ of a holomorphic function defined in a neighbourhood of the origin $O$ has a homogeneous expansion as an infinite sum of homogeneous polynomials, $g=P_{0}+P_{1}+\cdots$, with $\operatorname{deg} P_{j}=j$ (or $P_{j} \equiv 0$ ); the least $j \geq 0$ such that $P_{j}$ is not identically zero is the $\operatorname{order} \nu(g)$ of $g$. Let $f$ be a germ of a holomorphic self-map of $\mathbb{C}^{2}$ fixing the origin and tangent to the identity. We can write $f_{1}=z+g$ and $f_{2}=w+h$. Set $l=\operatorname{gcd}(g, h)$, and write $g=l g^{\circ}$ and $h=l h^{o}$. The pure order of $f$ at the origin is $\nu_{o}(f, O)=\min \left\{\nu\left(g^{o}\right), \nu\left(h^{o}\right)\right\}$. We say that the origin is a singular point for $f$ if $\nu_{o}(f, O) \geq 1$. If $\operatorname{Fix}(f)$ has (at least) two (local) components intersecting at the origin, we say that the origin is a corner.

More generally, let $S$ be a subset of a complex 2-manifold $M$; we shall denote by $\operatorname{End}(M, S)$ the set of germs about $S$ of holomorphic self-maps of $M$ sending $S$ into itself. Let $f \in \operatorname{End}(M, S)$ be such that $\left.f\right|_{S}=\mathrm{id}_{S}$, where $S$ is a 1-dimensional submanifold of $M$, and assume that $d f$ acts as the identity on the normal bundle of $S$ in $M$. Then we can extend the definitions of pure order, corner, singular point, and dicritical point to any $p \in S$, simply by choosing a chart of $M$ centered at $p$ and considering the local expression of $f$.

Our main theorem can now be stated as follows:

Theorem 1.6. Let $S$ be a 1-dimensional submanifold of a complex 2-manifold $M$ and let $f \in \operatorname{End}(M, S)$ be such that $\left.f\right|_{S}=\mathrm{id}_{S}$. Assume that $d f$ acts as the identity on the normal bundle of $S$ in $M$ and let $f$ be tangential to $S$. If $p \in S$ is a singular point of $f$, not a corner, with $\nu_{o}(f, p)=1$ and $\operatorname{Ind}(f, S, p) \neq 0$, then there exist parabolic curves for $f$ in $p$.

An important application of this result is the following. Starting from a map $f \in \operatorname{End}\left(\mathbb{C}^{2}, O\right)$ tangent to the identity and blowing up the origin, we obtain an $\tilde{f} \in \operatorname{End}(M, S)$, where $S \cong \mathbb{P}^{1}$ is the exceptional divisor of the blow-up. It turns out that $\left.\tilde{f}\right|_{S}=\operatorname{id}_{S}$, and that $d \tilde{f}$ acts as the identity on the normal bundle of $S$ in $M$. Furthermore, if the origin is an isolated fixed point of $f$, then no point $p \in S$ is a corner, and $\tilde{f}$ is tangential to $S$ if and only if the origin is nondicritical for $f$. If the origin is dicritical for $f$, then all directions are characteristic, and there are parabolic curves tangent to all but a finite number of them; so we concentrate 
on the nondicritical case. Every nondegenerate characteristic direction of $f$ is a singular point for $\tilde{f}$, and every singular point of $\tilde{f}$ is a characteristic direction of $f$. Moreover, if $p \in S$ is not singular, then no infinite orbit can get arbitrarily close to $p$ (in particular, no infinite orbit can converge to $p$, and thus there can be no parabolic curves at $p$ ); therefore, from a dynamical point of view only singular points are interesting (for the proof of all these assertions see [2]).

Finally, we shall say that $f$ is regular along the characteristic direction $[v] \in \mathbb{P}^{1}$ if the pure order of $\tilde{f}$ at $[v]$ is one. This is just a technical condition almost always satisfied (for instance, it is satisfied by the map in Example 1.5). Here is the promised

Corollary 1.7. Let $f \in \operatorname{End}\left(\mathbb{C}^{2}, O\right)$ be tangent to the identity with the origin as a nondicritical isolated fixed point. Let $[v] \in \mathbb{P}^{1}$ be a characteristic direction of $f$ and assume $f$ is regular along $[v]$ with $\operatorname{Ind}\left(\tilde{f}, \mathbb{P}^{1},[v]\right) \neq 0$ (here we identify $\mathbb{P}^{1}$ with the exceptional divisor of the blow-up of the origin, and $\tilde{f}$ is the blow-up of $f$ ). Then there exist parabolic curves for $f$ tangent to $[v]$ at the origin.

\section{BLOW-UP}

Since blow-ups will be a fundamental tool in our study we recall some basic definitions, referring to [1] for details. Let $M$ be a complex 2-manifold, and let $p \in M$. The blow-up of $M$ in $p$ is the set $\tilde{M}=(M \backslash\{p\}) \cup \mathbb{P}\left(T_{p} M\right)$, together with the projection $\pi: \tilde{M} \rightarrow M$ given by $\left.\pi\right|_{M \backslash\{p\}}=\mathrm{id}_{M \backslash\{p\}}$ and $\left.\pi\right|_{\mathbb{P}_{\left(T_{p} M\right)}} \equiv p$. The set $S=\mathbb{P}\left(T_{p} M\right)=\pi^{-1}(p)$ is the exceptional divisor of the blow-up.

It is possible to endow $\tilde{M}$ with a structure of a 2-dimensional complex manifold. Fix a chart $\psi=\left(z_{1}, z_{2}\right): U \rightarrow \mathbb{C}^{2}$ of $M$ centered at $p$. For $j=1,2$, set

$$
U_{j}=\left(U \backslash\left\{z_{j}=0\right\}\right) \cup\left(S \backslash \operatorname{Ker}\left(\left.d z_{j}\right|_{p}\right)\right),
$$

and let $\chi_{j}: U_{j} \rightarrow \mathbb{C}^{2}$ be given by

$$
\chi_{j}(q)_{h}= \begin{cases}z_{j}(q) & \text { if } j=h \text { and } q \in U \backslash\left\{z_{j}=0\right\}, \\ \frac{z_{h}(q)}{z_{j}(q)} & \text { if } j \neq h \text { and } q \in U \backslash\left\{z_{j}=0\right\}, \\ \frac{d\left(z_{h}\right)_{p}(q)}{d\left(z_{j}\right)_{p}(q)} & \text { if } \left.j \neq h \text { and } q \in S \backslash \operatorname{Ker}\left(\left.d z_{j}\right|_{p}\right)\right), \\ 0 & \text { if } \left.j=h \text { and } q \in S \backslash \operatorname{Ker}\left(\left.d z_{j}\right|_{p}\right)\right) .\end{cases}
$$

Then the charts $\left(U_{j}, \chi_{j}\right)$, together with an atlas of $M \backslash\{p\}$, endow $\tilde{M}$ with a structure of a 2-dimensional complex manifold such that the projection $\pi$ is holomorphic everywhere and given by

$$
\left[\psi \circ \pi \circ \chi_{j}^{-1}(w)\right]_{h}= \begin{cases}w_{j} & \text { if } j=h, \\ w_{j} w_{h} & \text { if } j \neq h .\end{cases}
$$

Let $f \in \operatorname{End}(M, p)$ be such that $d f_{p}$ is invertible. Then (see [1]) there exists a unique map $\tilde{f} \in \operatorname{End}(\tilde{M}, S)$, the blow-up of $f$ at $p$, such that $\pi \circ \tilde{f}=f \circ \pi$. The action of $\tilde{f}$ on $S$ is induced by the action of $d f_{p}$ on $\mathbb{P}\left(T_{p} M\right)$; in particular, if $d f_{p}=$ id, then $\left.\tilde{f}\right|_{S}=\mathrm{id}_{S}$. Finally, if $R$ is a submanifold of $M$, then the proper transform of $R$ is $\tilde{R}=\overline{\pi^{-1}(R \backslash\{p\})}$. 


\section{REsidual INDEX}

Let us fix notation and definitions that we use in the paper. $\mathcal{O}_{2}$ denotes the ring of germs of holomorphic functions defined in a neighbourhood of the origin $O$ of $\mathbb{C}^{2}$. If $S$ is a 1-dimensional submanifold of a complex 2-manifold $M$, a chart $(U, \varphi)$ of $M$ about $p \in S$ is adapted to $S$ if $U \cap S=\varphi^{-1}(\{(z, w) \mid z=0\})$. Let $f \in \operatorname{End}(M, S)$ be such that $\left.f\right|_{S}=\mathrm{id}_{S}$ and assume that $d f$ acts as the identity on the normal bundle of $S$ in $M$. Then in an adapted chart $(U, \varphi)$ centered at $p \in S$ we can write

$$
\left\{\begin{array}{l}
f_{1}(z, w)=z+z^{\mu+2} \hat{g}(z, w) \\
f_{2}(z, w)=w+z^{\nu+1} \hat{h}(z, w)
\end{array}\right.
$$

for suitable $\hat{g}, \hat{h} \in \mathcal{O}_{2}$ and $\mu, \nu \in \mathbb{N} \cup\{\infty\}$, where $\mu=\infty$ (resp., $\nu=\infty$ ) means $\hat{g} \equiv 0$ (resp., $\hat{h} \equiv 0$ ), and where $z$ does not divide either $\hat{g}$ or $\hat{h}$. After having introduced the (locally defined) meromorphic function

$$
k(w)=\lim _{z \rightarrow 0} \frac{f_{1}(z, w)-z}{z\left(f_{2}(z, w)-w\right)}= \begin{cases}0 & \text { if } \mu>\nu, \\ (\hat{g} / \hat{h})(0, w) & \text { if } \mu=\nu, \\ \infty & \text { if } \mu<\nu,\end{cases}
$$

Abate in [2] called $f$ degenerate along $S$ if $k \equiv \infty$. This definition is well posed (i.e., does not depend on the adapted chart chosen); furthermore he defined the residual index of $f$ at $p$ along $S$ by setting

$$
\operatorname{lnd}(f, S, p)=\operatorname{Res}_{0}(k(w))
$$

again, it is independent of the adapted chart chosen, and we observe that it might be nonzero at singular points of $f$ only. See [2] and [3] (in particular the latter) for a more thorough and deeper discussion of residual indices and related topics.

\section{Dynamics}

In this section we give the proof of Theorem 1.6 and Corollary 1.7. First, we introduce some definitions. Let us consider $f \in \operatorname{End}\left(\mathbb{C}^{2}, O\right)$ tangent to the identity, and $[v] \in \mathbb{P}^{1}$ a characteristic direction for $f$.

Definition 4.1. We say that $f$ is regular along $[v]$ if $\nu_{o}(\tilde{f},[v])=1$, where $\tilde{f}$ is the blow-up of $f$ at the origin.

Remark 4.2. If $[v]$ is a nondegenerate characteristic direction for $f$, then $f$ is regular along $[v]$. Generally, the converse is not true.

Example 4.3. Let us consider a map $f \in \operatorname{End}\left(\mathbb{C}^{2}, O\right)$ of order 2,

$$
\left\{\begin{array}{l}
f_{1}(z, w)=z+a_{2,0} z^{2}+a_{1,1} z w+a_{0,2} w^{2}+\cdots \\
f_{2}(z, w)=w+b_{2,0} z^{2}+b_{1,1} z w+b_{0,2} w^{2}+b_{3,0} z^{3} \cdots .
\end{array}\right.
$$

We observe that $[v]=[1: 0]$ is a characteristic direction for $f$ if and only if $b_{2,0}=0$. In this case, $f$ is regular along $[1: 0]$ if it is nondegenerate or it is degenerate but $b_{3,0} \neq 0$.

We can finally start working. Let $S$ be a 1-dimensional submanifold of a complex 2-manifold $M$, and let $f$ be a germ about $S$ of a holomorphic self-map of $M$ such that $\left.f\right|_{S}=\mathrm{id}_{S}$. Assume that $d f$ acts as the identity on the normal bundle of $S$ in $M$, and assume that $f$ is tangential to $S$. Let $p \in S$ be a noncorner and a singular point, with $\nu_{o}(f, p)=1$ and $\operatorname{Ind}(f, S, p) \neq 0$. We want to prove that there exist 
parabolic curves for $f$ in $p$. We work in a chart adapted to $S$ and centered at $p$. Then we can write

$$
\left\{\begin{array}{l}
f_{1}(z, w)=z+z^{r} A_{1}(z, w), \\
f_{2}(z, w)=w+z^{r} B_{1}(z, w),
\end{array}\right.
$$

for suitable $A_{1}, B_{1} \in \mathcal{O}_{2}, r \in \mathbb{N}^{*}$, with $\min \left\{\nu\left(A_{1}\right), \nu\left(B_{1}\right)\right\}=1$ and $\operatorname{gcd}\left(A_{1}, B_{1}\right)=1$. Since $f$ is tangential to $S$, we must have $A_{1}(z, w)=z A_{0}(z, w)$ with $\nu\left(A_{0}\right) \geq 0$. Let

$$
\left\{\begin{array}{l}
A_{0}(z, w)=a_{0,0}+\left(a_{1,0} z+a_{0,1} w\right)+\left(a_{2,0} z^{2}+a_{1,1} z w+a_{0,2} w^{2}\right)+\cdots, \\
B_{1}(z, w)=\left(b_{1,0} z+b_{0,1} w\right)+\left(b_{2,0} z^{2}+b_{1,1} z w+b_{0,2} w^{2}\right)+\cdots,
\end{array}\right.
$$

be the homogeneous expansions of $A_{0}$ and $B_{1}$ in a series of homogeneous polynomials. Since $\operatorname{gcd}\left(A_{1}, B_{1}\right)=1, z$ does not divide $B_{1}(z, w)$; thus we have $b_{0, j} \neq 0$, for some $j \geq 1$. An easy calculation shows that

$$
\operatorname{Ind}(f, S, p)=\operatorname{Res}_{0}\left(\frac{A_{0}(0, w)}{B_{1}(0, w)}\right)=\operatorname{Res}_{0}\left(\frac{a_{0,0}+a_{0,1} w+a_{0,2} w^{2}+\cdots}{b_{0,1} w+b_{0,2} w^{2}+b_{0,3} w^{3}+\cdots}\right) .
$$

Set

$$
\begin{aligned}
m & :=\min \left\{h \in \mathbb{N}: a_{0, h} \neq 0\right\}, \\
n & :=\min \left\{j \in \mathbb{N}: b_{0, j} \neq 0\right\} .
\end{aligned}
$$

We observe that it must be $m<n$ because otherwise, by (4.2), Ind $(f, S, p)=0$.

Let us dispose of the easier cases first.

Theorem 4.4. Assume that either

(a) $m<n-1$, or

(b) $m=n-1$ and $\operatorname{lnd}(f, S, p) \neq n$, or

(c) $m=0, n=1$ and $\operatorname{Ind}(f, S, p)=1$.

Then there exist (at least) $r+m(r+1)$ parabolic curves for $f$ at the origin.

Proof. Let $n=1$. By (4.2) it follows that $\operatorname{Ind}(f, S, p)=a_{0,0}\left(b_{0,1}\right)^{-1}$.

Assume that $\operatorname{Ind}(f, S, p) \neq 0,1$. Then $[v]:=\left[1: b_{1,0}\left(a_{0,0}-b_{0,1}\right)^{-1}\right]$ is a nondegenerate characteristic direction of $f$. In fact, by (4.1), $[1: w]$ is a characteristic direction if and only if

$$
\left\{\begin{array}{l}
a_{0,0}=\lambda, \\
b_{1,0}+b_{0,1} w=\lambda w
\end{array}\right.
$$

which is satisfied by $w=b_{1,0}\left(a_{0,0}-b_{0,1}\right)^{-1}$ and with $a_{0,0} \neq 0$. Hence, we have $r$ parabolic curves for $f$ tangent to $[v]$ at the origin (cf. [9], [10]).

Assume now $\operatorname{Ind}(f, S, p)=1$. We can write (4.1) as

$$
\left\{\begin{array}{l}
f_{1}(z, w)=z+a_{0,0} z^{r+1}+O\left(z^{r+2}, z^{r+1} w\right) \\
f_{2}(z, w)=w\left[1+a_{0,0} z^{r}+O\left(z^{r+1}, z^{r} w\right)\right]+O\left(z^{r+1}\right),
\end{array}\right.
$$

with $a_{0,0} \neq 0$. Setting $Z=\alpha z$, where $\alpha^{r}=-a_{0,0}$, we reduce to

$$
\left\{\begin{array}{l}
f_{1}(z, w)=z-z^{r+1}+O\left(z^{r+2}, z^{r+1} w\right), \\
f_{2}(z, w)=w\left[1-z^{r}+O\left(z^{r+1}, z^{r} w\right)\right]+O\left(z^{r+1}\right) .
\end{array}\right.
$$

The existence of $r$ parabolic curves at the origin for a map of this form is then a consequence of the results of [9, adapted as in [10 if $r>1$. We observe that these curves are tangent to $[0: 1]$ if $b_{1,0} \neq 0$, otherwise to [1:0].

Let $n>1$, and assume $m<n-1$, or otherwise $m=n-1$ and $\operatorname{Ind}(f, S, p) \neq n$. 
If $m=0, f$ has a nondegenerate characteristic direction. In fact, the system defining characteristic directions becomes

$$
\left\{\begin{array}{l}
a_{0,0}=\lambda, \\
b_{1,0}=\lambda w,
\end{array}\right.
$$

with $\lambda \neq 0$. Then, again from [9] and [10, we have the existence of $r$ parabolic curves for $f$ tangent to $\left[1: b_{1,0} / a_{0,0}\right]$ at the origin.

If $m \geq 1$ we know that

$$
\left\{\begin{array}{lr}
a_{0,0}=\cdots=a_{0, m-1}=0, & a_{0, m} \neq 0, \\
b_{0,1}=\cdots=b_{0, m}=\cdots=b_{0, n-1}=0, & b_{0, n} \neq 0 .
\end{array}\right.
$$

Let us consider the linear chain starting in $p \in S$ as defined in 5. It is a finite sequence of projective lines intersecting each other transversally and at most in one point, obtained by a finite sequence of blow-ups reducing corners to simpler singular points. Finiteness, as the possibility to obtain corners in a simpler form, is assured by the reduction theorem [2]. For our purposes it is sufficient to consider only a part of this linear chain. Blowing-up $f$ in $p$, let us denote by $\tilde{f}^{[1]}$ its blow-up, by $S_{1}$ the exceptional divisor, and by $\tau(p)$ the intersection point between $S_{1}$ and $\tilde{S}$, the proper transform of $S$.

Notation. To avoid heavy notation, at any step of this construction we will continue to denote by $S$ the proper transform $\tilde{S}$.

The second step consists in blowing-up $\tilde{f}^{[1]}$ in $\tau(p)$ and denoting by $\tilde{f}^{[2]}$ its blowup, by $S_{2}$ the exceptional divisor, and by $\tau(\tau(p))=\tau^{2}(p)$ the intersection point between $S_{2}$ and $S$. For $k \leq m$, iterating this process, we can define $\tilde{f}^{[k]}$ as the blow-up of $\tilde{f}^{[k-1]}$ in $\tau^{k-1}(p), S_{k}$ the exceptional divisor and $\tau^{k}(p)$ the intersection point between $S_{k}$ and $S$. We observe that this point is strictly related to the order of the chain, as defined in [5]. By induction, it is easy to check that, in a chart centered at $\tau^{k}(p)$, we have

$$
\left\{\begin{array}{c}
\tilde{f}_{1}^{[k]}(z, w)=z+z^{r} w^{k(r+1)-1}\left[-k b_{1,0} z^{2}+\left(a_{0, m}-k b_{0, m+1}\right) z w^{m-k+1}\right. \\
\left.+O\left(z w^{2}, z w^{m-k+2}\right)\right], \\
\tilde{f}_{2}^{[k]}(z, w)=w+z^{r} w^{k(r+1)-1}\left[b_{1,0} z w+b_{0, m+1} w^{m-k+2}+O\left(z w^{2}, w^{m-k+3}\right)\right] .
\end{array}\right.
$$

Let $z_{0}:=\left[a_{0, m}-(m+1) b_{0, m+1}\right](m+1)^{-1} b_{1,0}^{-1}$. We remark that $z_{0} \neq 0$. We claim that $\left[z_{0}: 1\right]$ is a nondegenerate characteristic direction for $\tilde{f}^{[m]}$. In fact, there exists $\lambda \in \mathbb{C} \backslash\{0\}$ such that

$$
\left\{\begin{array}{l}
-m b_{1,0} z_{0}^{r+2}+\left(a_{0, m}-m b_{0, m+1}\right) z_{0}^{r+1}=\lambda z_{0}, \\
b_{1,0} z_{0}^{r+1}+b_{0, m+1} z_{0}^{r}=\lambda
\end{array}\right.
$$

an easy computation shows that it suffices to choose

$$
\lambda= \begin{cases}b_{1,0} z_{0}^{r+1} & \text { if } m<n-1 \\ \frac{a_{0, n-1}}{n} z_{0}^{r} & \text { if } m=n-1\end{cases}
$$

So, again from $\left[9\right.$ and [10], there exist $\nu\left(\tilde{f}^{[m]}\right)-1=r+m(r+1)$ parabolic curves for $\tilde{f}^{[m]}$ tangent to $\left[z_{0}: 1\right]$ at the origin. Notice that a parabolic curve for $\tilde{f}^{[m]}$ cannot intersect the exceptional divisor since all points of the curve are attracted to the origin. Therefore, the push-forward of a parabolic curve for $\tilde{f}^{[m]}$ is a parabolic 
curve for $\tilde{f}^{[m-1]}$ tangent to $[0: 1]$ at the origin. The iteration of this process will give us $r+m(r+1)$ parabolic curves for $f$ tangent to [0:1] at the origin.

Remark 4.5. If $m=n-1$ and $\operatorname{Ind}(f, S, p)=n$, then $z_{0}=0$, and $\left[z_{0}: 1\right]$ is a degenerate characteristic direction for $\tilde{f}^{[n-1]}$. We can say much more: in fact $\left[z_{0}: 1\right]$ coincides with $\tau^{n}(p)$, and it is an irreducible singular point of type $\left(\star_{2}\right)$ for $\tilde{f}^{[n]}$, and thus the order of the linear chain, starting at $p$, is exactly $n$ (see [2] and [5] for the terminology). Therefore we cannot expect anything from the previous construction, because it ends at such a point.

Let us now deal with the hardest case. We shall prove the following:

Theorem 4.6. Let $n \geq 2$. If $m=n-1$ and $\operatorname{Ind}(f, S, p)=n$, then there exists (at least) one parabolic curve for $f$ at the origin.

First, we need another set of results.

Assume then $n \geq 2, m=n-1$ and $\operatorname{Ind}(f, S, p)=\frac{a_{0, n-1}}{b_{0, n}}=n$. We can write (4.1) as follows:

$$
\left\{\begin{aligned}
f_{1}(z, w)= & z+a_{0, n-1} z^{r+1} w^{n-1}+a_{1,0} z^{r+2}+O\left(z^{r+3}, z^{r+2} w, z^{r+1} w^{n}\right) \\
f_{2}(z, w)= & w\left[1+b_{0, n} z^{r} w^{n-1}+b_{1,1} z^{r+1}+O\left(z^{r+2}, z^{r+1} w, z^{r} w^{n}\right)\right] \\
& +b_{1,0} z^{r+1}+O\left(z^{r+2}\right)
\end{aligned}\right.
$$

with $r \in \mathbb{N}^{*}$ and $b_{1,0} \neq 0$, because $\nu_{o}(f, p)=1$.

In what follows we need to consider analytic changes of variables, defined in a neighbourhood of the origin $O \in \mathbb{C}^{2}$, and which involve roots and logarithms of the first complex variable $z$. This makes sense if $z$ belongs to a simply connected open set with $z=0$ on the boundary; for example, the $z$-plane with a cut. In this case, we fix, once and for all, one branch of the roots and the logarithm of $z$ in a way that $t=(\log z)^{\frac{1}{n}}$ belongs to an open sector of the $t$-plane with a vertex at the origin and a positive central angle not exceeding $\frac{\pi}{n}$.

Proposition 4.7. Let $\left(f_{1}, f_{2}\right)$ be a map of the form (4.5). Then we can choose local coordinates, defined in a suitable simply connected open set with $O$ on the boundary, relative to which the map takes the form:

(1) if $n=2$ :

$$
\left\{\begin{aligned}
\hat{f}_{1}(z, w)=z & -a^{-1} z^{r+1} w-z^{r+1+\frac{1}{2}}(\log z)^{\frac{1}{2}} \\
& +O\left(z^{r+2} \log z, z^{r+1+\frac{1}{2}} w(\log z)^{\frac{1}{2}}, z^{r+1} w^{2}\right) \\
\hat{f}_{2}(z, w)=w & {\left[1-\frac{a^{-1}}{2} z^{r} w-\frac{1}{2} z^{r+\frac{1}{2}}(\log z)^{\frac{1}{2}}+\frac{1}{2} \frac{z^{r+\frac{1}{2}}}{(\log z)^{\frac{1}{2}}}\right.} \\
& \left.+O\left(z^{r+1} \log z, z^{r+\frac{1}{2}} w(\log z)^{\frac{1}{2}}, z^{r} w^{2}\right)\right]+z^{r+1+\frac{1}{2}} \psi_{1}(z),
\end{aligned}\right.
$$

for some $a \neq 0$; 
(2) if $n \geq 3$ :

$$
\left\{\begin{aligned}
\hat{f}_{1}(z, w)=z- & (n-1) a^{-1} z^{r+1+\frac{n-2}{n}} w(\log z)^{\frac{n-2}{n}}-z^{r+1+\frac{n-1}{n}}(\log z)^{\frac{n-1}{n}} \\
+ & O\left(z^{r+2} \log z, z^{r+1+\frac{n-1}{n}} w(\log z)^{\frac{n-1}{n}}\right) \\
& +O\left(z^{r+1+\frac{n-3}{n}} w^{2}(\log z)^{\frac{n-3}{n}}, \ldots, z^{r+1} w^{n-1}\right) \\
\hat{f}_{2}(z, w)=w\left[1-\frac{n-1}{n} a^{-1} z^{r+\frac{n-2}{n}} w(\log z)^{\frac{n-2}{n}}\right. & \\
& -\frac{1}{n} z^{r+\frac{n-1}{n}}(\log z)^{\frac{n-1}{n}}+\frac{n-1}{n} \frac{z^{r+\frac{n-1}{n}}}{(\log z)^{\frac{1}{n}}} \\
+ & O\left(z^{r+1} \log z, z^{r+\frac{n-1}{n}} w(\log z)^{\frac{n-1}{n}}\right) \\
+ & \left.O\left(z^{r+\frac{n-3}{n}} w^{2}(\log z)^{\frac{n-3}{n}}, \ldots, z^{r} w^{n-1}\right)\right]+z^{r+1+\frac{1}{n}} \psi_{1}(z),
\end{aligned}\right.
$$

for some $a \neq 0$.

Furthermore, in both cases $\hat{f}_{1}, \hat{f}_{2}$ and $\psi_{1}(z)$ are analytic in $z,(\log z)^{\frac{1}{n}}$ and $w$, with $\psi_{1}$ of the form

$$
\psi_{1}(z)=\sum_{j=1}^{\infty} z^{\frac{j-1}{n}} R_{j}^{1}\left((\log z)^{\frac{1}{n}}\right)
$$

where $R_{j}^{1}(t)$ are analytic functions on the image, under the transformation $t=$ $(\log z)^{\frac{1}{n}}$, of our suitable simply connected open set with $z=0$ on its boundary.

Proof. Let $n \geq 2$. In the coordinates $(Z, W)$, with

$$
\left\{\begin{aligned}
Z & =\alpha z \\
W & =w
\end{aligned}\right.
$$

and $\alpha \neq 0$ an arbitrary parameter, (4.5) becomes

$$
\left\{\begin{aligned}
\bar{f}_{1}(Z, W)=Z & +a_{0, n-1} \alpha^{-r} Z^{r+1} W^{n-1}+a_{1,0} \alpha^{-r-1} Z^{r+2} \\
& +O\left(Z^{r+3}, Z^{r+2} W, Z^{r+1} W^{n}\right) \\
\bar{f}_{2}(Z, W)=W & {\left[1+b_{0, n} \alpha^{-r} Z^{r} W^{n-1}+b_{1,1} \alpha^{-r-1} Z^{r+1}\right.} \\
& \left.+O\left(Z^{r+2}, Z^{r+1} W, Z^{r} W^{n}\right)\right]+b_{1,0} \alpha^{-r-1} Z^{r+1}+O\left(Z^{r+2}\right) .
\end{aligned}\right.
$$

If $n=2$, let us consider the following change of variables:

$$
\left\{\begin{array}{l}
u=Z \\
v=W-a Z^{\frac{1}{2}}(\log Z)^{\frac{1}{2}},
\end{array}\right.
$$


with $a \neq 0$ an arbitrary parameter. Then (4.9) assumes the following form:

$$
\left\{\begin{aligned}
\hat{f}_{1}(u, v)=u+ & a_{0,1} \alpha^{-r} u^{r+1} v+a a_{0,1} \alpha^{-r} u^{r+1+\frac{1}{2}}(\log u)^{\frac{1}{2}} \\
& +O\left(u^{r+2} \log u, u^{r+1+\frac{1}{2}} v(\log u)^{\frac{1}{2}}, u^{r+1} v^{2}\right) \\
\hat{f}_{2}(u, v)=v\left[1+b_{0,2} \alpha^{-r} u^{r} v+a b_{0,2} \alpha^{-r} u^{r+\frac{1}{2}}(\log u)^{\frac{1}{2}}-\frac{a a_{0,1} \alpha^{-r}}{2} \frac{u^{r+\frac{1}{2}}}{(\log u)^{\frac{1}{2}}}\right. & \\
+ & \left.O\left(u^{r+1} \log u, u^{r+\frac{1}{2}} v(\log u)^{\frac{1}{2}}, u^{r} v^{2}\right)\right] \\
& +\alpha^{-r}\left(b_{1,0} \alpha^{-1}-a^{2} \frac{a_{0,1}}{2}\right) u^{r+1}+a^{2} \alpha^{-r}\left(b_{0,2}-\frac{a_{0,1}}{2}\right) u^{r+1} \log u \\
& +O\left(u^{r+1+\frac{1}{2}}(\log u)^{1+\frac{1}{2}}\right) .
\end{aligned}\right.
$$

Since $a_{0,1}=2 b_{0,2}$, if we choose $(\alpha, a)$ as a solution of

$$
\left\{\begin{array}{l}
b_{1,0} \alpha^{-1}-a^{2} 2^{-1} a_{0,1}=0 \\
a a_{0,1} \alpha^{-r}=-1
\end{array}\right.
$$

we obtain (4.6). It is not difficult to check that changes of variables of type (4.8) and (4.10) generate in $\hat{f}_{2}(u, 0)$ terms of the types

$$
\left\{\begin{array}{lll}
u^{r+1+\frac{1}{2}}(\log u)^{1+\frac{1}{2}}, & u^{r+1+\frac{1}{2}}(\log u)^{\frac{1}{2}}, & \frac{u^{r+1+\frac{1}{2}}}{(\log u)^{\frac{1}{2}}}, \\
u^{r+2}(\log u)^{2}, \quad u^{r+2} \log u, \quad u^{r+2}, & \\
u^{r+2+\frac{1}{2}}(\log u)^{1+\frac{1}{2}}, \quad u^{r+2+\frac{1}{2}}(\log u)^{\frac{1}{2}}, & \frac{u^{r+2+\frac{1}{2}}}{(\log u)^{\frac{1}{2}}}, \\
u^{r+3}(\log u)^{2}, \quad u^{r+3} \log u, \quad u^{r+3}, \ldots &
\end{array}\right.
$$

Then we can write

$$
\begin{aligned}
\hat{f}_{2}(u, 0)= & O\left(u^{r+1+\frac{1}{2}}(\log u)^{1+\frac{1}{2}}\right)=u^{r+1+\frac{1}{2}}\left[R_{1}^{1}\left((\log u)^{\frac{1}{2}}\right)+u^{\frac{1}{2}} R_{2}^{1}\left((\log u)^{\frac{1}{2}}\right)\right. \\
& \left.+u R_{3}^{1}\left((\log u)^{\frac{1}{2}}\right)+u^{1+\frac{1}{2}} R_{4}^{1}\left((\log u)^{\frac{1}{2}}\right)+\cdots\right]=u^{r+1+\frac{1}{2}} \psi_{1}(u)
\end{aligned}
$$

where $R_{j}^{1}(t)$ are analytic functions on the image, under the transformation $t=$ $(\log u)^{\frac{1}{2}}$ of our suitable simply connected open set with $u=0$ on its boundary.

Let $n \geq 3$. If we consider the change of coordinates

$$
\left\{\begin{array}{l}
u=Z, \\
v=W-a Z^{\frac{1}{n}}(\log Z)^{\frac{1}{n}},
\end{array}\right.
$$


with $a$ such that $a^{n}=\left(\alpha b_{0, n}\right)^{-1} b_{1,0}$, then (4.9) becomes

$$
\left\{\begin{aligned}
\hat{f}_{1}(u, v)= & +(n-1) a^{n-2} a_{0, n-1} \alpha^{-r} u^{r+1+\frac{n-2}{n}} v(\log u)^{\frac{n-2}{n}} \\
& +a^{n-1} a_{0, n-1} \alpha^{-r} u^{r+1+\frac{n-1}{n}}(\log u)^{\frac{n-1}{n}} \\
& +O\left(u^{r+2} \log u, u^{r+1+\frac{n-1}{n}} v(\log u)^{\frac{n-1}{n}}\right) \\
& +O\left(u^{r+1+\frac{n-3}{n}} v^{2}(\log u)^{\frac{n-3}{n}}, u^{r+1+\frac{n-4}{n}} v^{3}(\log u)^{\frac{n-4}{n}}, \ldots, u^{r+1} v^{n-1}\right), \\
\hat{f}_{2}(u, v)= & v\left[1+(n-1) a^{n-2} b_{0, n} \alpha^{-r} u^{r+\frac{n-2}{n}} v(\log u)^{\frac{n-2}{n}}\right. \\
& +a^{n-1} b_{0, n} \alpha^{-r} u^{r+\frac{n-1}{n}}(\log u)^{\frac{n-1}{n}}-\frac{n-1}{n} a^{n-1} a_{0, n-1} \alpha^{-r} \frac{u^{r+\frac{n-1}{n}}}{(\log u)^{\frac{1}{n}}} \\
& +O\left(u^{r+1} \log u, u^{r+\frac{n-1}{n}} v(\log u)^{\frac{n-1}{n}}\right) \\
& \left.+O\left(u^{r+\frac{n-3}{n}} v^{2}(\log u)^{\frac{n-3}{n}}, u^{r+\frac{n-4}{n}} v^{3}(\log u)^{\frac{n-4}{n}}, \ldots, u^{r} v^{n-1}\right)\right] \\
& +O\left(u^{r+1+\frac{1}{n}}(\log u)^{1+\frac{1}{n}}\right) .
\end{aligned}\right.
$$

Since $a_{0, n-1}=n b_{0, n}$, choosing $(\alpha, a)$ as a solution of

$$
\left\{\begin{array}{l}
b_{1,0} \alpha^{-1}-a^{n} n^{-1} a_{0, n-1}=0 \\
a^{n-1} a_{0, n-1} \alpha^{-r}=-1
\end{array}\right.
$$

we obtain (4.7).

As before, analyzing pure terms in $\hat{f}_{2}(u, 0)$ we have

$$
\begin{aligned}
\hat{f}_{2}(u, 0)= & O\left(u^{r+1+\frac{1}{n}}(\log u)^{1+\frac{1}{n}}\right)=u^{r+1+\frac{1}{n}}\left[R_{1}^{1}\left((\log u)^{\frac{1}{n}}\right)+u^{\frac{1}{n}} R_{2}^{1}\left((\log u)^{\frac{1}{n}}\right)\right. \\
& \left.+u^{\frac{2}{n}} R_{3}^{1}\left((\log u)^{\frac{1}{n}}\right)+\cdots+u R_{n+1}^{1}\left((\log u)^{\frac{1}{n}}\right)+\cdots\right]=u^{r+1+\frac{1}{n}} \psi_{1}(u),
\end{aligned}
$$

where $R_{j}^{1}(t)$ are analytic functions on the image, under the transformation $t=$ $(\log u)^{\frac{1}{n}}$ of our suitable simply connected open set with $u=0$ on its boundary.

Remark 4.8. In particular, for any $j \geq 1$ we can write $R_{j}^{1}(t)$ in the following form:

$$
R_{j}^{1}(t):=\sum_{k=1}^{\infty} \frac{c_{j,-k}^{1}}{t^{k}}+c_{j, 0}^{1}+c_{j, 1}^{1} t+\cdots+c_{j, m_{j}^{1}}^{1} t^{m_{j}^{1}}=: S_{j}^{1}(t)+P_{j}^{1}(t),
$$

for suitable $c_{j, k}^{1} \in \mathbb{C}$ and with $P_{j}^{1}(t):=c_{j, 0}^{1}+c_{j, 1}^{1} t+\cdots+c_{j, m_{j}^{1}}^{1} t^{m_{j}^{1}}$ such that $\operatorname{deg} P_{j}^{1}=m_{j}^{1}$ or $P_{j}^{1}(t) \equiv 0$. We shall say that $R_{j}^{1}$ satisfies the star property.

Proposition 4.9. Let $\left(\hat{f}_{1}, \hat{f}_{2}\right)$ be a map of the form (4.6), if $n=2$, or (4.7), if $n \geq 3$. Then there exists a sequence $\left\{Q_{h}(t)\right\}_{h \in \mathbb{N}}$ of functions analytic on the image, under the transformation $t=(\log z)^{\frac{1}{n}}$, of our suitable simply connected open set with $z=0$ on its boundary, such that if $w_{h+1}(z)$ is defined by

$$
\left\{\begin{array}{l}
w_{1}(z)=z^{\frac{2}{n}} Q_{0}\left((\log z)^{\frac{1}{n}}\right), \\
w_{h+1}(z)=w_{h}(z)+z^{\frac{h+2}{n}} Q_{h}\left((\log z)^{\frac{1}{n}}\right), \quad \text { if } h \geq 1,
\end{array}\right.
$$

then

$$
\hat{f}_{2}\left(z, w_{h+1}(z)\right)-w_{h+1}\left(\hat{f}_{1}\left(z, w_{h+1}(z)\right)\right)=z^{r+1+\frac{h+2}{n}} \psi_{h+2}(z),
$$


where $\psi_{h+2}$ is of the form $\psi_{h+2}(z)=\sum_{j=1}^{\infty} z^{\frac{j-1}{n}} R_{j}^{h+2}\left((\log z)^{\frac{1}{n}}\right)$, with $R_{j}^{h+2}(t)$ analytic functions on the image, under the transformation $t=(\log z)^{\frac{1}{n}}$, of our suitable simply connected open set with $z=0$ on its boundary.

Proof. In both cases we know that

$$
\psi_{1}(z)=R_{1}^{1}\left((\log z)^{\frac{1}{n}}\right)+z^{\frac{1}{n}} R_{2}^{1}\left((\log z)^{\frac{1}{n}}\right)+\cdots+z R_{n+1}^{1}\left((\log z)^{\frac{1}{n}}\right)+\cdots,
$$

where $R_{j}^{1}(t)$ are analytic functions on the image, under the transformation $t=$ $(\log z)^{\frac{1}{n}}$ of our suitable simply connected open set with $z=0$ on its boundary.

We prove the proposition by induction on $h$.

Let $h=0$. If we define $w_{1}(z):=z^{\frac{2}{n}} Q_{0}\left((\log z)^{\frac{1}{n}}\right)$, with $Q_{0}(t)$ a holomorphic solution of the differential equation

$$
t^{-(n-1)} Q^{\prime}(t)+(n-1)\left(1+\frac{1}{t^{n}}\right) Q(t)=-n t^{-(n-1)} R_{1}^{1}(t),
$$

we have

$$
\hat{f}_{2}\left(z, w_{1}(z)\right)-w_{1}\left(\hat{f}_{1}\left(z, w_{1}(z)\right)\right)=z^{r+1+\frac{2}{n}} \psi_{2}(z)
$$

with

$$
\psi_{2}(z)=R_{1}^{2}\left((\log z)^{\frac{1}{n}}\right)+z^{\frac{1}{n}} R_{2}^{2}\left((\log z)^{\frac{1}{n}}\right)+\cdots+z R_{n+1}^{2}\left((\log z)^{\frac{1}{n}}\right)+\cdots,
$$

and where $R_{j}^{2}(t)$ are analytic functions on the image, under the transformation $t=(\log z)^{\frac{1}{n}}$, of our suitable simply connected open set with $z=0$ on its boundary.

We study the differential equation (4.12).

First, an easy computation shows that there exists a unique formal solution of the following form:

$$
F_{0}(t):=\sum_{k=1}^{\infty} \frac{d_{-k}^{0}}{t^{k}}+d_{0}^{0}+d_{1}^{0} t+\cdots+d_{l_{0}}^{0} t^{l_{0}}
$$

with $T^{0}(t):=d_{0}^{0}+d_{1}^{0} t+\cdots+d_{l_{0}}^{0} t^{l_{0}}$, such that $\operatorname{deg} T^{0}=l_{0}$ or $T^{0}(t) \equiv 0$.

According to [13] (Theorem 12.1, p. 57) there exists, for sufficiently large $t$ in an open sector $S$ with vertex at the origin and a positive central angle not exceeding $\frac{\pi}{n}$, a holomorphic solution $Q_{0}(t)$ of (4.12), which is asymptotic to the formal solution $F_{0}(t)$, in every proper subsector $S^{\prime}$ of $S$. This means that for all $m \geq 1$

$$
\lim _{t \rightarrow \infty} t^{m}\left[Q_{0}(t)-\left(\sum_{k=1}^{m} \frac{d_{-k}^{0}}{t^{k}}+d_{0}^{0}+d_{1}^{0} t+\cdots+d_{l_{0}}^{0} t^{l_{0}}\right)\right]=0
$$

as $t$ tends to $\infty$ in $S^{\prime}$, and we write $Q_{0}(t) \sim F_{0}(t)$ for short.

Then (4.11) is satisfied for $h=0$.

Moreover, using Theorems 8.2, 8.3 and 8.8 of [13] it follows that $R_{1}^{2}(t)$ is asymptotic, in every proper subsector $S^{\prime}$ of $S$, to

$$
\sum_{k=1}^{\infty} \frac{c_{1,-k}^{2}}{t^{k}}+c_{1,0}^{2}+c_{1,1}^{2} t+\cdots+c_{1, m_{1}^{2}}^{2} t^{m_{1}^{2}}=: S_{1}^{2}(t)+P_{1}^{2}(t),
$$

for suitable $c_{1, k}^{2} \in \mathbb{C}$ and with $P_{1}^{2}(t):=c_{1,0}^{2}+c_{1,1}^{2} t+\cdots+c_{1, m_{1}^{2}}^{2} t^{m_{1}^{2}}$ such that $\operatorname{deg} P_{1}^{2}=m_{1}^{2}$ or $P_{1}^{2}(t) \equiv 0$. 
For $h>0$, one sees by induction that if (4.11) is satisfied by $w_{h}$, 4.11) is then satisfied by $w_{h+1}(z)=w_{h}(z)+z^{\frac{h+2}{n}} Q_{h}\left((\log z)^{\frac{1}{n}}\right)$ for a function $Q_{h}$, if and only if $Q_{h}$ is a solution of the differential equation

$$
t^{-(n-1)} Q^{\prime}(t)+(n-1)\left[(h+1)+\frac{1}{t^{n}}\right] Q(t)=-n t^{-(n-1)} R_{1}^{h+1}(t) .
$$

We observe that if $R_{1}^{h}$ and $Q_{h-1}$ are asymptotic to some functions which satisfy the star property, then also $R_{1}^{h+1}$ is asymptotic to a function which satisfies the same property, for all $h \geq 1$. Then, by the inductive hypothesis we can suppose that $R_{1}^{h+1}(t)$ is asymptotic to one function which satisfies the star property; hence

$$
R_{1}^{h+1}(t) \sim \sum_{k=1}^{\infty} \frac{c_{1,-k}^{h+1}}{t^{k}}+c_{1,0}^{h+1}+c_{1,1}^{h+1} t+\cdots+c_{1, m_{1}^{h+1}}^{h+1} t^{m_{1}^{h+1}}=: S_{1}^{h+1}(t)+P_{1}^{h+1}(t),
$$

for suitable $c_{1, k}^{h+1} \in \mathbb{C}$ and with $P_{1}^{h+1}(t):=c_{1,0}^{h+1}+c_{1,1}^{h+1} t+\cdots+c_{1, m_{1}^{h+1}}^{h+1} t^{m_{1}^{h+1}}$ such that $\operatorname{deg} P_{1}^{h+1}=m_{1}^{h+1}$ or $P_{1}^{h+1}(t) \equiv 0$. Also in this case it is possible to prove that there exists a unique formal solution of the differential equation (4.14) of the form

$$
F_{h}(t):=\sum_{k=1}^{\infty} \frac{d_{-k}^{h}}{t^{k}}+d_{0}^{h}+d_{1}^{h} t+\cdots+d_{l_{h}}^{h} t^{l_{h}},
$$

with $T^{h}(t):=d_{0}^{h}+d_{1}^{h} t+\cdots+d_{l_{h}}^{h} t^{l_{h}}$ such that $\operatorname{deg} T^{h}=l_{h}$ or $T^{h}(t) \equiv 0$.

According to 13 . (Theorem 12.1, p. 57) there exists, for sufficiently large $t$ in a suitable open sector $S$ with a vertex at the origin and a positive central angle not exceeding $\frac{\pi}{n}$, a holomorphic solution $Q_{h}(t)$ of (4.14) which is asymptotic to the formal solution $F_{h}(t)$, in every proper subsector $S^{\prime}$ of $S$. In particular, this means that for all $m \geq 1$

$$
Q_{h}(t)=\sum_{k=1}^{m} \frac{d_{-k}^{h}}{t^{k}}+d_{0}^{h}+d_{1}^{h} t+\cdots+d_{l_{h}}^{h} t^{l_{h}}+O\left(\frac{1}{t^{m}}\right),
$$

as $t$ tends to $\infty$ in $S^{\prime}$.

Corollary 4.10. Let $\left(\hat{f}_{1}, \hat{f}_{2}\right)$ be a map of the form (4.6), if $n=2$, or (4.7), if $n \geq 3$. Then for every $h \in \mathbb{N}$, we can choose local coordinates relative to which the map takes the form:

(1) if $n=2$ :

$$
\left\{\begin{aligned}
\hat{f}_{1}(Z, W)=Z & -a^{-1} Z^{r+1} W-Z^{r+1+\frac{1}{2}}(\log Z)^{\frac{1}{2}} \\
& +O\left(Z^{r+2}(\log Z)^{i}, Z^{r+1+\frac{1}{2}} W(\log Z)^{\frac{1}{2}}, Z^{r+1} W^{2}\right), \\
\hat{f}_{2}(Z, W)=W & {\left[1-\frac{a^{-1}}{2} Z^{r} W-\frac{1}{2} Z^{r+\frac{1}{2}}(\log Z)^{\frac{1}{2}}+\frac{1}{2} \frac{Z^{r+\frac{1}{2}}}{(\log Z)^{\frac{1}{2}}}\right.} \\
+ & \left.O\left(Z^{r+1}(\log Z)^{i}, Z^{r+\frac{1}{2}} W(\log Z)^{\frac{1}{2}}, Z^{r} W^{2}\right)\right] \\
+ & Z^{r+1+\frac{h+2}{2}} \psi_{h+2}(Z),
\end{aligned}\right.
$$

for some $i \geq 1$, where $\hat{f}_{1}, \hat{f}_{2}$ and $\psi_{h+2}(Z)$ are analytic in $Z,(\log Z)^{\frac{1}{2}}$, and $W$, with $Z$ belonging to our suitable simply connected open set with $Z=0$ on the boundary; 
(2) if $n \geq 3$ :

$$
\left\{\begin{aligned}
\hat{f}_{1}(Z, W)=Z & -(n-1) a^{-1} Z^{r+1+\frac{n-2}{n}} W(\log Z)^{\frac{n-2}{n}}-Z^{r+1+\frac{n-1}{n}}(\log Z)^{\frac{n-1}{n}} \\
& +O\left(Z^{r+2}(\log Z)^{i}, Z^{r+1+\frac{n-1}{n}} W(\log Z)^{\frac{n-1}{n}}\right) \\
& +O\left(Z^{r+1+\frac{n-3}{n}} W^{2}(\log Z)^{\frac{n-3}{n}}, \ldots, Z^{r+1} W^{n-1}\right), \\
\hat{f}_{2}(Z, W)=W & {\left[1-\frac{n-1}{n} a^{-1} Z^{r+\frac{n-2}{n}} W(\log Z)^{\frac{n-2}{n}}-\frac{1}{n} Z^{r+\frac{n-1}{n}}(\log Z)^{\frac{n-1}{n}}\right.} \\
& +\frac{n-1}{n} \frac{Z^{r+\frac{n-1}{n}}}{(\log Z)^{\frac{1}{n}}}+O\left(Z^{r+1}(\log Z)^{i}, Z^{r+\frac{n-1}{n}} W(\log Z)^{\frac{n-1}{n}}\right) \\
& \left.+O\left(Z^{r+\frac{n-3}{n}} W^{2}(\log Z)^{\frac{n-3}{n}}, \ldots, Z^{r} W^{n-1}\right)\right] \\
& +Z^{r+1+\frac{n+2}{n}} \psi_{h+2}(Z),
\end{aligned}\right.
$$

for some $i \geq 1$, where $\hat{f}_{1}, \hat{f}_{2}$ and $\psi_{h+2}(Z)$ are analytic in $Z,(\log Z)^{\frac{1}{n}}$, and $W$, with $Z$ belonging to our suitable simply connected open set with $Z=0$ on the boundary.

Proof. Let $w_{h+1}$ be given by Proposition 4.9, One has just to take as new coordinates

$$
\left\{\begin{array}{l}
Z=z, \\
W=w-w_{h+1}(z) .
\end{array}\right.
$$

We remark that $i=\max \{1, I / n\}$, where $I$ is the maximum power of $t$ appearing in the following estimate, which is obtained from (4.13) and is satisfied for all $m \geq 1$ and for sufficiently large $t$,

$$
Q_{0}(t)=\sum_{k=1}^{m} \frac{d_{-k}^{0}}{t^{k}}+d_{0}^{0}+d_{1}^{0} t+\cdots+d_{l_{0}}^{0} t^{l_{0}}+O\left(\frac{1}{t^{m}}\right) .
$$

Hence $I=l_{0}$ or $I=-\min \left\{k \geq 1 \mid d_{-k}^{0} \neq 0\right\}$.

By Corollary 4.10, we can choose local coordinates defined in our suitable simply connected open set with $O$ on the boundary, such that for every $h \in \mathbb{N}$, the map $\hat{f}$ is written as in (4.16), if $n=2$ or (4.17), if $n \geq 3$. Let us consider $h=2 n-3$; for our purposes it will be enough.

Now set

$$
D_{r+\frac{n-1}{n}, \delta}:=\left\{\zeta \in \mathbb{C} \quad|\quad| \zeta^{r+\frac{n-1}{n}}(\log \zeta)^{\frac{n-1}{n}}-\delta \mid<\delta\right\},
$$

for some $\delta>0$.

For an arbitrary $\delta$, this set has a number of connected components which depends on $r$ and $n$ : if $r$ is even, $D_{r+\frac{n-1}{n}, \delta}$ has $r+1$ connected and simply connected components with the origin on the boundary; if $r$ is odd but $n$ is not too big, the number of the connected components with the origin on the boundary is $r$; otherwise if $n$ is big enough this number is $r+1$.

Instead, if $\delta$ is small enough, the number of the components with the origin on the boundary is equal to $r+1$, in all cases, and the picture of the set looks like a flower, in which not all the petals have the same size. 
On these components, and for $\delta$ sufficiently small, the functions $w_{h}$ are well defined.

Let us fix some notation. Put

$$
\mathcal{E}_{n}^{\beta}(\delta):=\left\{w \in \operatorname{Hol}\left(D_{r+\frac{n-1}{n}, \delta}, \mathbb{C}\right) \quad \mid \quad w(\zeta)=\zeta^{2}(\log \zeta)^{\beta} h^{o}(\zeta), \quad\left\|h^{o}\right\|_{\infty}<\infty\right\},
$$

with $\beta \in \mathbb{R}$. It is a Banach space with the norm $\|w\|_{\mathcal{E}_{n}^{\beta}(\delta)}=\left\|h^{o}\right\|_{\infty}$. For $w \in \mathcal{E}_{n}^{\beta}(\delta)$, put $\hat{f}^{w}(\zeta)=\hat{f}_{1}(\zeta, w(\zeta))$.

Assume we have found $\hat{w} \in \mathcal{E}_{n}^{\beta}(\delta)$ which satisfies the following properties:

(i) for all $\zeta \in D_{r+\frac{n-1}{n}, \delta}$

$$
\hat{w}\left(\hat{f}_{1}(\zeta, \hat{w}(\zeta))\right)=\hat{f}_{2}(\zeta, \hat{w}(\zeta))
$$

(ii) there exists a positive constant $\delta_{0}$ such that, if $0<\delta<\delta_{0}$, then $\hat{f}^{\hat{w}}$ sends every component of $D_{r+\frac{n-1}{n}, \delta}$ into itself and $\hat{\zeta}_{k} \rightarrow 0$ as $k \rightarrow \infty$, where $\hat{\zeta}_{k}$ denotes the sequence of an iterate of an arbitrary point $\zeta \in D_{r+\frac{n-1}{n}, \delta}$ by the transformation $\hat{f} \hat{w}(\zeta):=\hat{\zeta}_{1}$;

then the restriction of $\hat{\varphi}(\zeta):=(\zeta, \hat{w}(\zeta))$ to any component of $D_{r+\frac{n-1}{n}, \delta}$ is a parabolic curve for $\hat{f}$. In fact, equation (4.18) implies that $\hat{\varphi}\left(D_{r+\frac{n-1}{n}, \delta}\right)$ is invariant under $\hat{f}$ and property (ii) implies that $\left(\left.\hat{f}\right|_{\hat{\varphi}\left(D_{r+\frac{n-1}{n}, \delta}\right)}\right)^{k} \rightarrow O$, as $k \rightarrow \infty$.

First, we must find a solution of (4.18). The function $\hat{w}$ will be obtained as a fixed point of a functional operator $T$, which acts as a contraction on a suitable closed convex subset of $\mathcal{E}_{n}^{\beta}(\delta)$ and which we are going to describe.

If $\hat{f}$ is given by (4.16) or (4.17) we denote $z_{1}:=\hat{f}_{1}(z, w)$ and $w_{1}:=\hat{f}_{2}(z, w)$. Suppose $z, z_{1}$ belong to the same connected component of $D_{r+\frac{n-1}{n}, \delta}$ and define

$$
H(z, w)=w-\frac{z^{\frac{1}{n}}}{z_{1}^{\frac{1}{n}}} w_{1} .
$$

A direct computation shows that:

(1) if $n=2$

$$
H(z, w)=O\left(\frac{z^{r+\frac{1}{2}}}{(\log z)^{\frac{1}{2}}} w, z^{r+\frac{1}{2}} w^{2}(\log z)^{\frac{1}{2}}, z^{r+2+\frac{1}{2}}(\log z)^{J}\right),
$$

(2) if $n \geq 3$

$$
\begin{aligned}
H(z, w) & =O\left(\frac{z^{r+\frac{n-1}{n}}}{(\log z)^{\frac{1}{n}}} w, z^{r+\frac{n-1}{n}} w^{2}(\log z)^{\frac{n-1}{n}}, z^{r+2+\frac{n-1}{n}}(\log z)^{J}\right) \\
& +O\left(z^{r+\frac{n-3}{n}} w^{3}(\log z)^{\frac{n-3}{n}}, z^{r+\frac{n-4}{n}} w^{4}(\log z)^{\frac{n-4}{n}}, \ldots, z^{r} w^{n}\right)
\end{aligned}
$$

where, in both cases, $J$ is the maximum power of $\log z$ appearing in (4.15), the asymptotic expansion of $R_{1}^{2 n-1}\left((\log z)^{\frac{1}{n}}\right)$.

Remark 4.11. In particular, if $\operatorname{deg} P_{1}^{2 n-1}=m_{1}^{2 n-1}$, then $J=m_{1}^{2 n-1} / n$; otherwise if $P_{1}^{2 n-1} \equiv 0$, then $J=-\min \left\{k \geq 1 \mid c_{1,-k}^{2 n-1} \neq 0\right\} / n$. 
Let us take $\beta=|J|$, and let $T$ be the operator on $\mathcal{E}_{n}^{|J|}(\delta)$ defined by

$$
T w\left(\zeta_{0}\right)=\zeta_{0}^{\frac{1}{n}} \sum_{k=0}^{\infty} \zeta_{k}^{-\frac{1}{n}} H\left(\zeta_{k}, w\left(\zeta_{k}\right)\right)
$$

where $\zeta_{k}:=\left(\hat{f}^{w}\right)^{k}\left(\zeta_{0}\right)$. In the remainder of this section, we shall prove that $T$ is well defined for any $\zeta_{0} \in D_{r+\frac{n-1}{n}, \delta}$ and for $\delta$ sufficiently small, and that $T$, restricted to a suitable closed convex subset of $\mathcal{E}_{n}^{|J|}(\delta)$, is contracting, so that it has a fixed point. Assume that this is done, and let $\tilde{w}$ be the fixed point. Then $\tilde{w}=\hat{w}$, since it is not difficult to see that $\tilde{w}$ is a fixed point of $T$ if and only if it satisfies (4.18). We show the implication $(\Rightarrow)$ : let $\zeta_{0} \in D_{r+\frac{n-1}{n}, \delta}$; then

$$
\begin{aligned}
\tilde{w}\left(\zeta_{1}\right) & =T \tilde{w}\left(\zeta_{1}\right)=\zeta_{1}^{\frac{1}{n}} \sum_{k=1}^{\infty} \zeta_{k}^{-\frac{1}{n}} H\left(\zeta_{k}, \tilde{w}\left(\zeta_{k}\right)\right) \\
& =\frac{\zeta_{1}^{\frac{1}{n}}}{\zeta_{0}^{\frac{1}{n}}}\left(\zeta_{0}^{\frac{1}{n}} \sum_{k=0}^{\infty} \zeta_{k}^{-\frac{1}{n}} H\left(\zeta_{k}, \tilde{w}\left(\zeta_{k}\right)\right)-H\left(\zeta_{0}, \tilde{w}\left(\zeta_{0}\right)\right)\right) \\
& =\frac{\zeta_{1}^{\frac{1}{n}}}{\zeta_{0}^{\frac{1}{n}}}\left[T \tilde{w}\left(\zeta_{0}\right)-H\left(\zeta_{0}, \tilde{w}\left(\zeta_{0}\right)\right)\right]=\frac{\zeta_{1}^{\frac{1}{n}}}{\zeta_{0}^{\frac{1}{n}}}\left[\tilde{w}\left(\zeta_{0}\right)-\tilde{w}\left(\zeta_{0}\right)+\frac{\zeta_{0}^{\frac{1}{n}}}{\zeta_{1}^{\frac{1}{n}}} \hat{f}_{2}\left(\zeta_{0}, \tilde{w}\left(\zeta_{0}\right)\right)\right] \\
& =\hat{f}_{2}\left(\zeta_{0}, \tilde{w}\left(\zeta_{0}\right)\right) .
\end{aligned}
$$

The next results establish some useful bounds. In particular, the next lemma shows that property (ii) is satisfied for each $w \in \mathcal{E}_{n}^{|J|}(\delta)$, if $\delta$ is chosen small enough and if $\left\|h^{o}\right\|_{\infty} \leq 1$.

Lemma 4.12. Let $\hat{f}$ be a map of the form (4.16) or (4.17), with $h=2 n-3$. For each $w \in \mathcal{E}_{n}^{|J|}(\delta)$ such that $\left\|h^{o}\right\|_{\infty} \leq 1$, let $\left\{\zeta_{k}\right\}$ be the sequence of iterates of $\zeta$ by the transformation $\hat{f}^{w}(\zeta)=\hat{f}_{1}(\zeta, w(\zeta))$. Then there is a positive constant $\delta_{0}$ such that if $\zeta$ belongs to a connected component of $D_{r+\frac{n-1}{n}, \delta_{0}}$, then $\zeta_{k}$ belongs to the same component of $D_{r+\frac{n-1}{n}, \delta_{0}}$ for every $k \in \mathbb{N}$, and

$$
\begin{aligned}
\frac{2}{3} \frac{|\zeta|^{r+\frac{n-1}{n}}|\log \zeta|^{\frac{n-1}{n}}}{\left|1+k\left(r+\frac{n-1}{n}\right) \zeta^{r+\frac{n-1}{n}}(\log \zeta)^{\frac{n-1}{n}}\right|} & \leq\left|\zeta_{k}\right|^{r+\frac{n-1}{n}}\left|\log \zeta_{k}\right|^{\frac{n-1}{n}} \\
& \leq 2 \frac{|\zeta|^{r+\frac{n-1}{n}}|\log \zeta|^{\frac{n-1}{n}}}{\left|1+k\left(r+\frac{n-1}{n}\right) \zeta^{r+\frac{n-1}{n}}(\log \zeta)^{\frac{n-1}{n}}\right|}
\end{aligned}
$$

Proof. From the hypothesis on $w(\zeta)$, we have

$$
\frac{1}{\zeta_{1}^{r+\frac{n-1}{n}}\left(\log \zeta_{1}\right)^{\frac{n-1}{n}}}=\frac{1}{\zeta^{r+\frac{n-1}{n}}(\log \zeta)^{\frac{n-1}{n}}}+r+\frac{n-1}{n}+g(\zeta),
$$

where $g(\zeta)=O\left(\frac{1}{\log \zeta}\right)=o(1)$. Observe that $\zeta$ and $\zeta_{1}$ are in the same component, i.e. the desired component is invariant under $\hat{f}$. Moreover, $g(\zeta)$ represents a function bounded by $K \frac{1}{|\log \zeta|}$, where $K$ is a constant independent of $w$. Iterating and dividing by $k$, we get

$$
\frac{1}{k \zeta_{k}^{r+\frac{n-1}{n}}\left(\log \zeta_{k}\right)^{\frac{n-1}{n}}}=\frac{1}{k \zeta^{r+\frac{n-1}{n}}(\log \zeta)^{\frac{n-1}{n}}}+\left(r+\frac{n-1}{n}\right)+\frac{\sum_{j=0}^{k-1} g\left(\zeta_{j}\right)}{k}
$$


where $g_{k}(\zeta)=\frac{1}{k} \sum_{j=0}^{k-1} g\left(\zeta_{j}\right)$ converges to 0 when $k$ goes to $\infty$, by Cesàro convergence. So, with $\zeta \in D_{r+\frac{n-1}{n}, \delta}$ and $\delta$ sufficiently small, we have

$$
\left(r+\frac{n-1}{n}\right) \zeta_{k}^{r+\frac{n-1}{n}}\left(\log \zeta_{k}\right)^{\frac{n-1}{n}} \sim \frac{1}{k} .
$$

From (4.23) we have, for all $k \in \mathbb{N}$

$$
\begin{aligned}
\frac{1}{\zeta_{k}^{r+\frac{n-1}{n}}\left(\log \zeta_{k}\right)^{\frac{n-1}{n}}}= & \frac{1}{\zeta^{r+\frac{n-1}{n}}(\log \zeta)^{\frac{n-1}{n}}}\left(1+k\left(r+\frac{n-1}{n}\right) \zeta^{r+\frac{n-1}{n}}(\log \zeta)^{\frac{n-1}{n}}\right) \\
& \cdot\left[1+\frac{\zeta^{r+\frac{n-1}{n}}(\log \zeta)^{\frac{n-1}{n}} \sum_{j=0}^{k-1} g\left(\zeta_{j}\right)}{1+k\left(r+\frac{n-1}{n}\right) \zeta^{r+\frac{n-1}{n}}(\log \zeta)^{\frac{n-1}{n}}}\right]
\end{aligned}
$$

where $\sum_{j=0}^{k-1} g\left(\zeta_{j}\right)=o(k)$. Hence, for $\zeta \rightarrow 0$

$$
\frac{\zeta^{r+\frac{n-1}{n}}(\log \zeta)^{\frac{n-1}{n}} \sum_{j=0}^{k-1} g\left(\zeta_{j}\right)}{1+k\left(r+\frac{n-1}{n}\right) \zeta^{r+\frac{n-1}{n}}(\log \zeta)^{\frac{n-1}{n}}} \rightarrow 0,
$$

and the quantities inside the brackets in (4.25) are uniformly close to 1 ; so for $\delta$ small enough we get the inequalities of (4.22).

Corollary 4.13. Let $\left\{\zeta_{k}\right\}$ and $\delta=\delta_{0}$ be defined as in Lemma 4.12, with $w \in \mathcal{E}_{n}^{|J|}(\delta)$ such that $\left\|h^{o}\right\|_{\infty} \leq 1$. Then for each real number $s>r+\frac{n-1}{n}$, for each $q \geq 0$, there exists a constant $C_{s, q}$ such that, for every $\zeta \in D_{r+\frac{n-1}{n}, \delta}$, we have

$$
\sum_{k=0}^{\infty}\left|\zeta_{k}\right|^{s}\left|\log \zeta_{k}\right|^{q} \leq C_{s, q}|\zeta|^{s-\left(r+\frac{n-1}{n}\right)}|\log | \zeta||^{q-\frac{n-1}{n}} .
$$

Proof. Let $\zeta \in D_{r+\frac{n-1}{n}, \delta}$. From the first inequality of (4.22) we get, for all $k \in \mathbb{N}$

$$
\frac{2}{3} \frac{|\zeta|^{r+\frac{n-1}{n}}}{\left|1+k\left(r+\frac{n-1}{n}\right) \zeta^{r+\frac{n-1}{n}}(\log \zeta)^{\frac{n-1}{n}}\right|} \frac{1}{\left|\log \zeta_{k}\right|^{\frac{n-1}{n}}} \leq\left|\zeta_{k}\right|^{r+\frac{n-1}{n}},
$$

and for $\delta$ small enough

$$
\log \left(\frac{2|\zeta|^{r+\frac{n-1}{n}}\left|\log \zeta_{k}\right|^{\frac{1-n}{n}}}{3\left|1+k\left(r+\frac{n-1}{n}\right) \zeta^{r+\frac{n-1}{n}}(\log \zeta)^{\frac{n-1}{n}}\right|}\right) \leq\left(r+\frac{n-1}{n}\right) \log \left|\zeta_{k}\right|<0 .
$$

Taking the modulus, we get

$$
\begin{aligned}
\left(r+\frac{n-1}{n}\right) \mid & \log \left|\zeta_{k}\right| \mid \\
\leq & \left|\log \left(\frac{2}{3} \frac{|\zeta|^{r+\frac{n-1}{n}}}{\left|1+k\left(r+\frac{n-1}{n}\right) \zeta^{r+\frac{n-1}{n}}(\log \zeta)^{\frac{n-1}{n}}\right|} \frac{1}{\left|\log \zeta_{k}\right|^{\frac{n-1}{n}}}\right)\right| \\
\leq & \left|\log \left(\frac{2}{3} \frac{|\zeta|^{r+\frac{n-1}{n}}}{\left|1+k\left(r+\frac{n-1}{n}\right) \zeta^{r+\frac{n-1}{n}}(\log \zeta)^{\frac{n-1}{n}}\right|}\right)\right| \\
& +\frac{n-1}{n}|\log | \log \zeta_{k}|| .
\end{aligned}
$$


Since $\log |\log z| \leq|\log | z||$, for $z$ sufficiently close to 0 , the last inequality becomes $(4.26)$

$$
\begin{aligned}
r|\log | \zeta_{k}|| & \leq\left|\log \left(\frac{2}{3} \frac{|\zeta|^{r+\frac{n-1}{n}}}{\left|1+k\left(r+\frac{n-1}{n}\right) \zeta^{r+\frac{n-1}{n}}(\log \zeta)^{\frac{n-1}{n}}\right|}\right)\right| \\
& \leq\left(r+\frac{n-1}{n}\right)|\log | \zeta||+\left|\log \frac{\left|1+k\left(r+\frac{n-1}{n}\right) \zeta^{r+\frac{n-1}{n}}(\log \zeta)^{\frac{n-1}{n}}\right|}{\frac{2}{3}}\right| .
\end{aligned}
$$

Since $\zeta \in D_{r+\frac{n-1}{n}, \delta}$ then $\operatorname{Re}\left(\zeta^{r+\frac{n-1}{n}}(\log \zeta)^{\frac{n-1}{n}}\right)>0$, and we get

$$
\begin{aligned}
0 & <\log \frac{\left|1+k\left(r+\frac{n-1}{n}\right) \zeta^{r+\frac{n-1}{n}}(\log \zeta)^{\frac{n-1}{n}}\right|}{\frac{2}{3}} \\
& \leq \log \frac{1+\left|k\left(r+\frac{n-1}{n}\right) \zeta^{r+\frac{n-1}{n}}(\log \zeta)^{\frac{n-1}{n}}\right|}{\frac{2}{3}} .
\end{aligned}
$$

Therefore, we can write (4.26) as

$$
|\log | \zeta_{k}|| \leq \frac{1}{r}\left[\left(r+\frac{n-1}{n}\right)|\log | \zeta||+\log \frac{1+\left|k\left(r+\frac{n-1}{n}\right) \zeta^{r+\frac{n-1}{n}}(\log \zeta)^{\frac{n-1}{n}}\right|}{\frac{2}{3}}\right] .
$$

From the second inequality of (4.22) we have, for all $k \in \mathbb{N}$

$$
\left|\zeta_{k}\right|^{r+\frac{n-1}{n}} \leq 2 \frac{|\zeta|^{r+\frac{n-1}{n}}}{\left|1+k\left(r+\frac{n-1}{n}\right) \zeta^{r+\frac{n-1}{n}}(\log \zeta)^{\frac{n-1}{n}}\right|} \frac{|\log \zeta|^{\frac{n-1}{n}}}{\left|\log \zeta_{k}\right|^{\frac{n-1}{n}}} .
$$

From (4.24) it follows that there exists a constant $C$ independent of $k$ and $\zeta$, such that $|\log \zeta| \leq C\left|\log \zeta_{k}\right|$, for all $k \in \mathbb{N}$. Hence we have, for all $s>0$ and $k \in \mathbb{N}$

$$
\left|\zeta_{k}\right|^{s} \leq C_{s} \frac{|\zeta|^{s}}{\left|1+k\left(r+\frac{n-1}{n}\right) \zeta^{r+\frac{n-1}{n}}(\log \zeta)^{\frac{n-1}{n}}\right|^{s\left(r+\frac{n-1}{n}\right)^{-1}}}
$$

for some constant $C_{s}$ depending only on $s$. Since $\operatorname{Re}\left(\zeta^{r+\frac{n-1}{n}}(\log \zeta)^{\frac{n-1}{n}}\right)>0$, we get

$$
\left|\zeta_{k}\right|^{s} \leq C_{s} \frac{|\zeta|^{s}}{\left(1+\left|k\left(r+\frac{n-1}{n}\right) \zeta^{r+\frac{n-1}{n}}(\log \zeta)^{\frac{n-1}{n}}\right|^{2}\right)^{\frac{s}{2}\left(r+\frac{n-1}{n}\right)^{-1}}} .
$$

Hence, there are constants $K_{1}, K_{s, q}$ and $K_{s, q}^{1}$ such that

$$
\begin{aligned}
& \sum_{k=0}^{\infty}\left|\zeta_{k}\right|^{s}\left|\log \zeta_{k}\right|^{q} \leq\left. K_{1} \sum_{k=0}^{\infty}\left|\zeta_{k}\right|^{s}|\log | \zeta_{k}\right|^{q} \\
& \leq K_{s, q}|\zeta|^{s} \sum_{k=0}^{\infty} \frac{\left[\left(r+\frac{n-1}{n}\right)|\log | \zeta||+\log \frac{1+\left|k\left(r+\frac{n-1}{n}\right) \zeta^{r+\frac{n-1}{n}}(\log \zeta)^{\frac{n-1}{n}}\right|}{\frac{2}{3}}\right]^{q}}{\left(1+\left|k\left(r+\frac{n-1}{n}\right) \zeta^{r+\frac{n-1}{n}}(\log \zeta)^{\frac{n-1}{n}}\right|^{2}\right)^{\frac{s}{2}\left(r+\frac{n-1}{n}\right)^{-1}}} \\
& \leq K_{s, q}^{1}|\zeta|^{s-\left(r+\frac{n-1}{n}\right)}|\log \zeta|^{-\frac{n-1}{n}} \int_{0}^{\infty} \frac{\left[\left(r+\frac{n-1}{n}\right)|\log | \zeta||+\log \frac{3(1+u)}{2}\right]^{q}}{\left(1+u^{2}\right)^{\frac{s}{2}\left(r+\frac{n-1}{n}\right)^{-1}}} d u .
\end{aligned}
$$

The last integral converges if and only if $s>r+\frac{n-1}{n}$, and it gives us a contribution of type $K_{s, q}^{2}|\log \zeta|^{q}$, for some constant $K_{s, q}^{2}$. 
Let us now study more carefully the operator $T$. From (4.19), or equivalently (4.20), and Corollary 4.13 we get the following estimate:

$$
|T w(\zeta)| \leq\left. C_{n, J}|\zeta|^{2}|\log | \zeta\right|^{|J|-\frac{n-1}{n}}<\left.|\zeta|^{2}|\log | \zeta\right|^{|J|} ;
$$

hence $T$ sends the unit ball of $\mathcal{E}_{n}^{|J|}(\delta)$ into itself. We will be able to prove more than this: $T$ sends into itself the convex closed set

$$
\mathcal{F}_{n}^{|J|}(\delta)=\left\{\left.w \in \mathcal{E}_{n}^{|J|}(\delta) \quad|\quad| w(\zeta)|\leq| \zeta\right|^{2}|\log | \zeta||^{|J|}, \quad\left|w^{\prime}(\zeta)\right| \leq|\zeta||\log | \zeta||^{|J|}\right\} .
$$

Then it will be enough to show that $T$ is a contraction on $\mathcal{F}_{n}^{|J|}(\delta)$. But first we need another set of results.

Lemma 4.14. Let $\left\{\zeta_{k}\right\}$ and $\delta=\delta_{0}$ be defined as in Lemma 4.12, with $w \in \mathcal{E}_{n}^{|J|}\left(\delta_{0}\right)$ such that $\left\|h^{o}\right\|_{\infty} \leq 1$. If $\left|w^{\prime}(\zeta)\right| \leq\left.|\zeta||\log | \zeta\right|^{|J|}$ in $D_{r+\frac{n-1}{n}, \delta_{0}}$, then there is a positive constant $\delta_{1}$ such that, for every $k \in \mathbb{N}$ and for every $\zeta \in \bar{D}_{r+\frac{n-1}{n}, \delta_{1}}$, we have the inequality

$$
\left|\frac{d \zeta_{k}}{d \zeta}\right| \leq c\left|\frac{\zeta_{k}}{\zeta}\right|^{r+1+\frac{n-1}{n}}\left|\frac{\log \zeta_{k}}{\log \zeta}\right|^{\frac{n-1}{n}}
$$

for some positive constant $c$.

Proof. Let us consider only the case $n \geq 3$, because if $n=2$ the computations are easier. By (4.17) we get

$$
\begin{aligned}
\frac{1}{\zeta_{1}^{r+\frac{n-1}{n}}\left(\log \zeta_{1}\right)^{\frac{n-1}{n}}}= & \frac{1}{\zeta^{r+\frac{n-1}{n}}(\log \zeta)^{\frac{n-1}{n}}}+r+\frac{n-1}{n}+\left(\frac{n-1}{n}\right) \frac{1}{\log \zeta} \\
& +O\left(\zeta^{\frac{1}{n}}(\log \zeta)^{i-\frac{n-1}{n}}, \frac{w(\zeta)}{\zeta^{\frac{1}{n}}(\log \zeta)^{\frac{1}{n}}}, \frac{w(\zeta)^{p-1}}{\zeta^{\frac{p-1}{n}}(\log \zeta)^{\frac{p-1}{n}}}\right)
\end{aligned}
$$

with $p$ an integer and $3 \leq p \leq n$. Our aim is to look for a function $g(\zeta)$ such that (4.28)

$$
\begin{aligned}
\frac{1}{\zeta_{1}^{r+\frac{n-1}{n}}\left(\log \zeta_{1}\right)^{\frac{n-1}{n}}}+g\left(\zeta_{1}\right)= & \frac{1}{\zeta^{r+\frac{n-1}{n}}(\log \zeta)^{\frac{n-1}{n}}}+r+\frac{n-1}{n}+g(\zeta) \\
& +O\left(\zeta^{\frac{1}{n}}(\log \zeta)^{i-\frac{n-1}{n}}, \frac{w(\zeta)}{\zeta^{\frac{1}{n}}(\log \zeta)^{\frac{1}{n}}}, \frac{w(\zeta)^{p-1}}{\zeta^{\frac{p-1}{n}}(\log \zeta)^{\frac{p-1}{n}}}\right)
\end{aligned}
$$

Comparing the previous equations we get

$$
g\left(\zeta_{1}\right)-g(\zeta)=\left(\frac{1-n}{n}\right) \frac{1}{\log \zeta}+O\left(\zeta^{\frac{1}{n}}(\log \zeta)^{i-\frac{n-1}{n}}, \frac{w(\zeta)}{\zeta^{\frac{1}{n}}(\log \zeta)^{\frac{1}{n}}}, \frac{w(\zeta)^{p-1}}{\zeta^{\frac{p-1}{n}}(\log \zeta)^{\frac{p-1}{n}}}\right) .
$$

The hypotheses on $w \in \mathcal{E}_{n}^{|J|}(\delta)$ and the approximation $g\left(\zeta_{1}\right)=g(\zeta)+g^{\prime}(\zeta)\left(\zeta_{1}-\zeta\right)$ $+O\left(\left(\zeta_{1}-\zeta\right)^{2}\right)$ imply that

$$
g^{\prime}(\zeta)\left(\zeta_{1}-\zeta\right)+O\left(\left(\zeta_{1}-\zeta\right)^{2}\right)=-\left(\frac{n-1}{n}\right) \frac{1}{\log \zeta}+O\left(\zeta^{\frac{1}{n}}(\log \zeta)^{i-\frac{n-1}{n}}\right) .
$$

From (4.17) we deduce that

$$
\zeta_{1}-\zeta=-\zeta^{r+1+\frac{n-1}{n}}(\log \zeta)^{\frac{n-1}{n}}+\zeta^{r+1+\frac{n-2}{n}} \phi(\zeta, w),
$$


with $\zeta^{r+1+\frac{n-2}{n}} \phi(\zeta, w(\zeta))=O\left(\zeta^{r+2}(\log \zeta)^{i}\right)$, and so we obtain

$$
\begin{aligned}
-\zeta^{r+1+\frac{n-1}{n}}(\log \zeta)^{\frac{n-1}{n}} g^{\prime}(\zeta) & +O\left(\zeta^{r+2}(\log \zeta)^{i}\right) g^{\prime}(\zeta) \\
& =-\left(\frac{n-1}{n}\right) \frac{1}{\log \zeta}+O\left(\zeta^{\frac{1}{n}}(\log \zeta)^{i-\frac{n-1}{n}}\right),
\end{aligned}
$$

and hence

$$
g^{\prime}(\zeta)=\left(\frac{n-1}{n}\right) \frac{1}{\zeta^{r+1+\frac{n-1}{n}}(\log \zeta)^{1+\frac{n-1}{n}}} .
$$

Let $g(\zeta)$ be a solution of the previous differential equation. Then (4.28) can be written as

$$
\frac{1}{\zeta_{1}^{r+\frac{n-1}{n}}\left(\log \zeta_{1}\right)^{\frac{n-1}{n}}}+g\left(\zeta_{1}\right)=\frac{1}{\zeta^{r+\frac{n-1}{n}}(\log \zeta)^{\frac{n-1}{n}}}+r+\frac{n-1}{n}+g(\zeta)+\varphi(\zeta, w(\zeta)),
$$

where $\varphi$ is a function holomorphic in $\{\zeta, w, \log \zeta\}$ and

$$
\varphi(\zeta, w(\zeta))=O\left(\zeta^{\frac{1}{n}}(\log \zeta)^{i-\frac{n-1}{n}}, \frac{w(\zeta)}{\zeta^{\frac{1}{n}}(\log \zeta)^{\frac{1}{n}}}, \frac{w(\zeta)^{p-1}}{\zeta^{\frac{p-1}{n}}(\log \zeta)^{\frac{p-1}{n}}}\right) .
$$

Now we want to estimate $\frac{d}{d \zeta} \varphi(\zeta, w(\zeta))$. The hypotheses on $w(\zeta)$ and $w^{\prime}(\zeta)$ imply that

$$
\begin{aligned}
\frac{d}{d \zeta}\left(\frac{w(\zeta)}{\zeta^{\frac{1}{n}}(\log \zeta)^{\frac{1}{n}}}\right) & =O\left(\frac{w^{\prime}(\zeta)}{\zeta^{\frac{1}{n}}(\log \zeta)^{\frac{1}{n}}}, \frac{w(\zeta)}{\zeta^{\frac{1}{n}+1}(\log \zeta)^{\frac{1}{n}}}\right)=O\left(\zeta^{\frac{n-1}{n}}(\log \zeta)^{|J|-\frac{1}{n}}\right) \\
\frac{d}{d \zeta}\left(\zeta^{\frac{1}{n}}(\log \zeta)^{i-\frac{n-1}{n}}\right) & =O\left(\zeta^{\frac{1}{n}-1}(\log \zeta)^{i-\frac{n-1}{n}}\right) \\
\frac{d}{d \zeta}\left(\frac{w(\zeta)^{p-1}}{\zeta^{\frac{p-1}{n}}(\log \zeta)^{\frac{p-1}{n}}}\right) & =O\left(\frac{w^{\prime}(\zeta) w(\zeta)^{p-2}}{\zeta^{\frac{p-1}{n}}(\log \zeta)^{\frac{p-1}{n}}}, \frac{w(\zeta)^{p-1}}{\zeta^{\frac{p-1}{n}+1}(\log \zeta)^{\frac{p-1}{n}}}\right) \\
& =O\left(\zeta^{2(p-1)-1-\frac{p-1}{n}}(\log \zeta)^{|J|(p-1)-\frac{p-1}{n}}\right) .
\end{aligned}
$$

Therefore

$$
\frac{d}{d \zeta} \varphi(\zeta, w(\zeta))=O\left(\zeta^{\frac{1}{n}-1}(\log \zeta)^{i-\frac{n-1}{n}}\right)
$$

Iterating (4.30) we have

$$
\begin{aligned}
\frac{1}{\zeta_{k}^{r+\frac{n-1}{n}}\left(\log \zeta_{k}\right)^{\frac{n-1}{n}}}+g\left(\zeta_{k}\right)=\frac{1}{\zeta^{r+\frac{n-1}{n}}(\log \zeta)^{\frac{n-1}{n}}} & +k\left(r+\frac{n-1}{n}\right)+g(\zeta) \\
& +\sum_{j=0}^{k-1} \varphi\left(\zeta_{j}, w\left(\zeta_{j}\right)\right),
\end{aligned}
$$

and differentiating in $\zeta$ this last relation, we get

$$
\begin{aligned}
-\left\{\frac{\left(r+\frac{n-1}{n}\right) \log \zeta_{k}+\frac{n-1}{n}}{\zeta_{k}^{r+1+\frac{n-1}{n}}\left(\log \zeta_{k}\right)^{1+\frac{n-1}{n}}}-g^{\prime}\left(\zeta_{k}\right)\right\} \frac{d \zeta_{k}}{d \zeta}= & -\left\{\frac{\left(r+\frac{n-1}{n}\right) \log \zeta+\frac{n-1}{n}}{\zeta^{r+1+\frac{n-1}{n}}(\log \zeta)^{1+\frac{n-1}{n}}}-g^{\prime}(\zeta)\right\} \\
& +\sum_{j=0}^{k-1} \frac{d}{d \zeta_{j}} \varphi\left(\zeta_{j}, w\left(\zeta_{j}\right)\right) \frac{d \zeta_{j}}{d \zeta}
\end{aligned}
$$


Replacing (4.29) in the previous expression, we have

$$
\begin{aligned}
-\left(r+\frac{n-1}{n}\right) \frac{1}{\zeta_{k}^{r+1+\frac{n-1}{n}}\left(\log \zeta_{k}\right)^{\frac{n-1}{n}}} \frac{d \zeta_{k}}{d \zeta}= & -\left(r+\frac{n-1}{n}\right) \frac{1}{\zeta^{r+1+\frac{n-1}{n}}(\log \zeta)^{\frac{n-1}{n}}} \\
& +\sum_{j=0}^{k-1} \frac{d}{d \zeta_{j}} \varphi\left(\zeta_{j}, w\left(\zeta_{j}\right)\right) \frac{d \zeta_{j}}{d \zeta}
\end{aligned}
$$

At this point we can proceed by induction on $k$. If $k=1$, then (4.32) becomes

$$
\frac{d \zeta_{1}}{d \zeta}=\frac{\zeta_{1}^{r+1+\frac{n-1}{n}}\left(\log \zeta_{1}\right)^{\frac{n-1}{n}}}{\zeta^{r+1+\frac{n-1}{n}}(\log \zeta)^{\frac{n-1}{n}}}\left\{1-\frac{n}{n r+n-1} \zeta^{r+1+\frac{n-1}{n}}(\log \zeta)^{\frac{n-1}{n}} \frac{d}{d \zeta} \varphi(\zeta, w(\zeta))\right\},
$$

and from (4.31) we deduce that

$$
\zeta^{r+1+\frac{n-1}{n}}(\log \zeta)^{\frac{n-1}{n}} \frac{d}{d \zeta} \varphi(\zeta, w(\zeta))=O\left(\zeta^{r+1}(\log \zeta)^{i}\right) .
$$

For $\delta$ small enough, the quantities inside the brackets are close to 1 , so there exists a constant $c$ such that

$$
\left|\frac{d \zeta_{1}}{d \zeta}\right| \leq c\left|\frac{\zeta_{1}}{\zeta}\right|^{r+1+\frac{n-1}{n}}\left|\frac{\log \zeta_{k}}{\log \zeta}\right|^{\frac{n-1}{n}}
$$

Let $k>1$. We can write (4.32) as

$$
\begin{aligned}
\frac{d \zeta_{k}}{d \zeta}=\{1 & \left.-\left(r+\frac{n-1}{n}\right)^{-1} \zeta^{r+1+\frac{n-1}{n}}(\log \zeta)^{\frac{n-1}{n}}\left(\sum_{j=0}^{k-1} \frac{d}{d \zeta_{j}} \varphi\left(\zeta_{j}, w\left(\zeta_{j}\right)\right) \frac{d \zeta_{j}}{d \zeta}\right)\right\} \\
& \cdot \frac{\zeta_{k}^{r+1+\frac{n-1}{n}}\left(\log \zeta_{k}\right)^{\frac{n-1}{n}}}{\zeta^{r+1+\frac{n-1}{n}}(\log \zeta)^{\frac{n-1}{n}}} .
\end{aligned}
$$

Using the inductive hypothesis, (4.31), and Corollary 4.13 we get

$$
\begin{aligned}
\sum_{j=0}^{k-1}\left|\frac{d}{d \zeta_{j}} \varphi\left(\zeta_{j}, w\left(\zeta_{j}\right)\right)\right|\left|\frac{d \zeta_{j}}{d \zeta}\right| & \leq \frac{C}{|\zeta|^{r+1+\frac{n-1}{n}}|\log \zeta|^{\frac{n-1}{n}}} \sum_{j=0}^{k-1}\left|\zeta_{j}\right|^{r+1}\left|\log \zeta_{j}\right|^{i} \\
& \leq \frac{C}{|\zeta|^{r+1+\frac{n-1}{n}}|\log \zeta|^{\frac{n-1}{n}}} \sum_{j=0}^{\infty}\left|\zeta_{j}\right|^{r+1}\left|\log \zeta_{j}\right|^{i} \\
& \leq C_{1} \frac{\left.|\zeta|^{\frac{1}{n}}|\log | \zeta\right|^{i-\frac{n-1}{n}}}{|\zeta|^{r+1+\frac{n-1}{n}}|\log \zeta|^{\frac{n-1}{n}}},
\end{aligned}
$$

for some constant $C$ and $C_{1}$. Hence

$$
\zeta^{r+1+\frac{n-1}{n}}(\log \zeta)^{\frac{n-1}{n}}\left(\sum_{j=0}^{k-1} \frac{d}{d \zeta_{j}} \varphi\left(\zeta_{j}, w\left(\zeta_{j}\right)\right) \frac{d \zeta_{j}}{d \zeta}\right)=O\left(\zeta^{\frac{1}{n}}(\log \zeta)^{i-\frac{n-1}{n}}\right)
$$

and the quantities inside the brackets in (4.33) are uniformly close to 1 , so we get (4.27). 
Lemma 4.15. Let $T$ be defined as in (4.21), and let $w \in \mathcal{E}_{n}^{|J|}(\delta)$ with $\left\|h^{o}\right\|_{\infty} \leq 1$. If $\left|w^{\prime}(\zeta)\right| \leq\left.|\zeta||\log | \zeta\right|^{|J|}$ for all $\zeta \in D_{r+\frac{n-1}{n}, \delta}$, then for $\delta$ small enough, we have

$$
\left|\frac{d}{d \zeta}(T w)(\zeta)\right| \leq|\zeta||\log | \zeta||^{|J|}, \quad \text { for all } \zeta \in D_{r+\frac{n-1}{n}, \delta} .
$$

Proof. Let us write

$$
\frac{d}{d \zeta}(T w)(\zeta)=\mathcal{S}_{1}+\mathcal{S}_{2}+\mathcal{S}_{3}
$$

We have to estimate the three terms $\mathcal{S}_{1}, \mathcal{S}_{2}$, and $\mathcal{S}_{3}$.

The term $\mathcal{S}_{1}$ comes from the differentiation of the term $\zeta^{\frac{1}{n}}$, i.e.

$$
\mathcal{S}_{1}=\frac{\zeta^{\frac{1}{n}-1}}{n} \sum_{k=0}^{\infty} \zeta_{k}^{-\frac{1}{n}} H\left(\zeta_{k}, w\left(\zeta_{k}\right)\right)
$$

Using (4.20) and Corollary 4.13, we get

$$
\left|\mathcal{S}_{1}\right| \leq \frac{1}{n|\zeta|^{\frac{n-1}{n}}} \sum_{k=0}^{\infty}\left|\zeta_{k}\right|^{r+2+\frac{n-2}{n}}\left|\log \zeta_{k}\right|^{|J|} \leq C_{1}|\zeta||\log | \zeta||^{|J|-\frac{n-1}{n}} \leq \frac{1}{3}|\zeta||\log | \zeta||^{|J|} .
$$

The term $\mathcal{S}_{2}$ is given by

$$
\mathcal{S}_{2}=\zeta^{\frac{1}{n}} \sum_{k=0}^{\infty} \zeta_{k}^{-\frac{1}{n}} \frac{\partial}{\partial w} H\left(\zeta_{k}, w\left(\zeta_{k}\right)\right) \frac{d w}{d \zeta_{k}} \frac{d \zeta_{k}}{d \zeta}
$$

From (4.20) and from the hypothesis on $w$, we have the following estimate:

$$
\left|\frac{\partial}{\partial w} H(\zeta, w(\zeta))\right| \leq C_{2} \frac{|\zeta|^{r+\frac{n-1}{n}}}{|\log \zeta|^{\frac{1}{n}}},
$$

for some constant $C_{2}$. Using this inequality, Lemma 4.14, and the hypothesis on $w^{\prime}(\zeta)$, we get

$$
\begin{aligned}
\left|\frac{\partial}{\partial w} H\left(\zeta_{k}, w\left(\zeta_{k}\right)\right) \frac{d w}{d \zeta_{k}} \frac{d \zeta_{k}}{d \zeta}\right| & \leq C_{3} \frac{\left|\zeta_{k}\right|^{r+\frac{n-1}{n}}}{\left|\log \zeta_{k}\right|^{\frac{1}{n}}}\left|\zeta_{k}\right||\log | \zeta_{k}||^{|J|}\left|\frac{\zeta_{k}}{\zeta}\right|^{r+1+\frac{n-1}{n}}\left|\frac{\log \zeta_{k}}{\log \zeta}\right|^{\frac{n-1}{n}} \\
& \leq C_{4} \frac{\left.\left|\zeta_{k}\right|^{2 r+2+2\left(\frac{n-1}{n}\right)}|\log | \zeta_{k}\right|^{|J|+\frac{n-2}{n}}}{\left.|\zeta|^{r+1+\frac{n-1}{n}}|\log | \zeta\right|^{\frac{n-1}{n}}}
\end{aligned}
$$

for some constants $C_{3}$ and $C_{4}$. Hence, using Corollary 4.13, we obtain

$$
\begin{aligned}
\left|\mathcal{S}_{2}\right| & \leq \frac{C_{4}}{\left.|\zeta|^{r+1+\frac{n-2}{n}}|\log | \zeta||\right|^{\frac{n-1}{n}}} \sum_{k=0}^{\infty}\left|\zeta_{k}\right|^{2 r+2+2\left(\frac{n-1}{n}\right)-\frac{1}{n}}|\log | \zeta_{k}||^{|J|+\frac{n-2}{n}} \\
& \leq C_{5}|\zeta||\log | \zeta||^{|J|-1} \leq \frac{1}{3}|\zeta||\log | \zeta||^{|J|}
\end{aligned}
$$

for some constant $C_{5}$.

The term $\mathcal{S}_{3}$ is given by

$$
\mathcal{S}_{3}=\zeta^{\frac{1}{n}} \sum_{k=0}^{\infty} \frac{\partial}{\partial \zeta_{k}}\left[\zeta_{k}^{-\frac{1}{n}} H\left(\zeta_{k}, w\left(\zeta_{k}\right)\right)\right] \frac{d \zeta_{k}}{d \zeta} .
$$

Let us define $u(\cdot)$ by the equality

$$
\mathcal{S}_{3}=\zeta^{\frac{1}{n}} \sum_{k=0}^{\infty} \zeta_{k}^{-\frac{1}{n}} u\left(\zeta_{k}\right)
$$


An easy computation shows that

$$
u\left(\zeta_{k}\right)=\left[-\frac{\zeta_{k}^{-1}}{n} H\left(\zeta_{k}, w\left(\zeta_{k}\right)\right)+\frac{\partial}{\partial \zeta_{k}} H\left(\zeta_{k}, w\left(\zeta_{k}\right)\right)\right] \frac{d \zeta_{k}}{d \zeta} .
$$

Since $H(\zeta, w(\zeta))=O\left(\zeta^{r+2+\frac{n-1}{n}}(\log \zeta)^{|J|}\right)$, we get

$$
\frac{\partial}{\partial \zeta} H(\zeta, w(\zeta))=O\left(\zeta^{r+1+\frac{n-1}{n}}(\log \zeta)^{|J|}\right),
$$

and hence

$$
\left|u\left(\zeta_{k}\right)\right| \leq C_{6}\left|\zeta_{k}\right|^{r+1+\frac{n-1}{n}}\left|\log \zeta_{k}\right|^{|J|}\left|\frac{d \zeta_{k}}{d \zeta}\right|,
$$

for some constant $C_{6}$ independent of $w$ and $w^{\prime}$. Using Lemma 4.14, we can proceed to a further estimate

$$
\left|u\left(\zeta_{k}\right)\right| \leq \frac{C_{7}}{|\zeta|^{r+1+\frac{n-1}{n}}|\log \zeta|^{\frac{n-1}{n}}}\left|\zeta_{k}\right|^{2 r+2+2\left(\frac{n-1}{n}\right)}\left|\log \zeta_{k}\right|^{|J|+\frac{n-1}{n}} .
$$

Finally, using Corollary 4.13 again, there exists a constant $C_{8}$ such that

$$
\begin{aligned}
\left|\mathcal{S}_{3}\right| & \leq \frac{C_{7}}{|\zeta|^{r+1+\frac{n-2}{n}}|\log \zeta|^{\frac{n-1}{n}}} \sum_{k=0}^{\infty}\left|\zeta_{k}\right|^{2 r+2+2\left(\frac{n-1}{n}\right)-\frac{1}{n}}\left|\log \zeta_{k}\right|^{|J|+\frac{n-1}{n}} \\
& \leq C_{8}|\zeta||\log | \zeta||^{|J|-\frac{n-1}{n}} \leq \frac{1}{3}|\zeta||\log | \zeta||^{|J|}
\end{aligned}
$$

Therefore, for $\delta$ small enough, we get, for all $\zeta \in D_{r+\frac{n-1}{n}, \delta}$,

$$
\left|\frac{d}{d \zeta}(T w)(\zeta)\right| \leq\left|\mathcal{S}_{1}\right|+\left|\mathcal{S}_{2}\right|+\left|\mathcal{S}_{3}\right| \leq|\zeta||\log | \zeta||^{|J|} .
$$

From this last lemma it follows that the operator $T$ sends the convex closed set $\mathcal{F}_{n}^{|J|}(\delta)$ into itself. It remains to prove that $T$ is a contraction. We need a last result.

Lemma 4.16. Let $\hat{f}$ be a map of the form (4.16) or (4.17), with $h=2 n-3$. Let $u(\zeta)=\zeta^{2}(\log \zeta)^{|J|} h_{1}(\zeta)$ and $v(\zeta)=\zeta^{2}\left(\left.\log \zeta\right|^{|J|} h_{2}(\zeta)\right.$ be two functions in $\mathcal{F}_{n}^{|J|}(\delta)$. Let $\left\{\zeta_{k}\right\}$ and $\left\{\zeta_{k}^{\prime}\right\}$ be, respectively, the iterates of $\zeta$ by $\hat{f}_{1}(\zeta, u(\zeta))$ and $\hat{f}_{1}(\zeta, v(\zeta))$. Then, for $\delta$ small enough, there exists a constant $K$ such that, for all $k \in \mathbb{N}$

$$
\left|\zeta_{k}^{\prime}-\zeta_{k}\right| \leq K|\zeta|^{3-\frac{1}{n}}|\log | \zeta||^{|J|-\frac{1}{n}}\left\|h_{2}-h_{1}\right\|_{\infty} .
$$

Proof. For $\zeta$ and $\zeta^{\prime}$ in the same connected component of $D_{r+\frac{n-1}{n}, \delta}$, we estimate the variation

$$
\hat{f}_{1}\left(\zeta^{\prime}, v\left(\zeta^{\prime}\right)\right)-\hat{f}_{1}(\zeta, u(\zeta))
$$

From (4.16) or (4.17), with $h=2 n-3$, we have

$$
\begin{aligned}
& \hat{f}_{1}\left(\zeta^{\prime}, v\left(\zeta^{\prime}\right)\right)-\hat{f}_{1}(\zeta, u(\zeta)) \\
& =\left[1-\frac{\left(\zeta^{\prime}\right)^{r+1+\frac{n-1}{n}}\left(\log \zeta^{\prime}\right)^{\frac{n-1}{n}}-\zeta^{r+1+\frac{n-1}{n}}(\log \zeta)^{\frac{n-1}{n}}}{\zeta^{\prime}-\zeta}+O\left(\left|\zeta^{\prime \prime}\right|^{r+1}\left|\log \zeta^{\prime \prime}\right|^{i}\right)\right] \\
& \cdot\left(\zeta^{\prime}-\zeta\right)+\left(v\left(\zeta^{\prime}\right)-u(\zeta)\right) O\left(\left|\zeta^{\prime \prime}\right|^{r+1+\frac{n-2}{n}}\left|\log \zeta^{\prime \prime}\right|^{\frac{n-2}{n}}\right)
\end{aligned}
$$


where $\left|\zeta^{\prime \prime}\right|=\max \left\{|\zeta|,\left|\zeta^{\prime}\right|\right\}$. We observe that when $k$ goes to infinity then $\frac{\zeta_{k}^{\prime}}{\zeta_{k}} \sim 1$ by (4.22), so we can replace $\left|\zeta^{\prime \prime}\right|$ by $|\zeta|$ in these estimates. Therefore

$$
\begin{aligned}
v\left(\zeta^{\prime}\right)-u(\zeta) & =v\left(\zeta^{\prime}\right)-v(\zeta)+v(\zeta)-u(\zeta) \\
& =O\left(\left.|\zeta||\log | \zeta\right|^{|J|}\right)\left(\zeta^{\prime}-\zeta\right)+O\left(\left.|\zeta|^{2}|\log | \zeta\right|^{|J|}\right)\left\|h_{2}-h_{1}\right\|_{\infty} .
\end{aligned}
$$

Using this last relation, (4.34) becomes

$$
\begin{aligned}
\zeta_{1}^{\prime}-\zeta_{1}= & \left(\zeta^{\prime}-\zeta\right)\left[1-\frac{\left(\zeta^{\prime}\right)^{r+1+\frac{n-1}{n}}\left(\log \zeta^{\prime}\right)^{\frac{n-1}{n}}-\zeta^{r+1+\frac{n-1}{n}}(\log \zeta)^{\frac{n-1}{n}}}{\zeta^{\prime}-\zeta}\right. \\
& \left.+O\left(|\zeta|^{r+1}|\log | \zeta||^{i}\right)\right] \\
& +O\left(|\zeta|^{\left.r+3+\frac{n-2}{n}|\log | \zeta||^{|J|+\frac{n-2}{n}}\right)\left\|h_{2}-h_{1}\right\|_{\infty} .}\right.
\end{aligned}
$$

For $\zeta$ and $\zeta^{\prime} \in D_{r+\frac{n-1}{n}, \delta}$, and $\delta$ small enough, the modulus of the quantity inside the brackets is bounded by 1 , and so we get

$$
\left|\zeta_{1}^{\prime}-\zeta_{1}\right| \leq\left|\zeta^{\prime}-\zeta\right|+K_{1}|\zeta|^{r+3+\frac{n-2}{n}}|\log | \zeta||^{|J|+\frac{n-2}{n}}\left\|h_{2}-h_{1}\right\|_{\infty},
$$

for some constant $K_{1}$. Iterating this process and taking $\zeta=\zeta^{\prime}$, we get, for all $k \in \mathbb{N}$

$$
\left|\zeta_{k}^{\prime}-\zeta_{k}\right| \leq\left. K_{1} \sum_{p=0}^{\infty}\left|\zeta_{p}\right|^{r+3+\frac{n-2}{n}}|\log | \zeta_{p}\right|^{|J|+\frac{n-2}{n}}\left\|h_{2}-h_{1}\right\|_{\infty}
$$

and by Corollary 4.13

$$
\left|\zeta_{k}^{\prime}-\zeta_{k}\right| \leq\left. K|\zeta|^{3-\frac{1}{n}}|\log | \zeta\right|^{|J|-\frac{1}{n}}\left\|h_{2}-h_{1}\right\|_{\infty},
$$

for some constant $K$.

Proposition 4.17. Let $T$ be defined as in (4.21). For $\delta$ small enough, the restriction of $T$ is a contraction of the convex closed subset $\mathcal{F}_{n}^{|J|}(\delta)$ of the Banach space $\mathcal{E}_{n}^{|J|}(\delta)$ into itself.

Proof. Let $u(\zeta)=\zeta^{2}(\log \zeta)^{|J|} h_{1}(\zeta)$ and $v(\zeta)=\zeta^{2}(\log \zeta)^{|J|} h_{2}(\zeta)$ be two functions in $\mathcal{F}_{n}^{|J|}(\delta)$. We can write the variation

$$
T u(\zeta)-T v(\zeta)=\zeta^{\frac{1}{n}} \sum_{k=0}^{\infty}\left[\zeta_{k}^{-\frac{1}{n}} H\left(\zeta_{k}, u\left(\zeta_{k}\right)\right)-\left(\zeta_{k}^{\prime}\right)^{-\frac{1}{n}} H\left(\zeta_{k}^{\prime}, v\left(\zeta_{k}^{\prime}\right)\right)\right]
$$

as the sum of two terms

$$
\mathcal{R}_{1}=\zeta^{\frac{1}{n}} \sum_{k=0}^{\infty} \zeta_{k}^{-\frac{1}{n}}\left[H\left(\zeta_{k}, u\left(\zeta_{k}\right)\right)-H\left(\zeta_{k}^{\prime}, v\left(\zeta_{k}^{\prime}\right)\right)\right]
$$

and

$$
\mathcal{R}_{2}=\zeta^{\frac{1}{n}} \sum_{k=0}^{\infty}\left[\zeta_{k}^{-\frac{1}{n}}-\left(\zeta_{k}^{\prime}\right)^{-\frac{1}{n}}\right] H\left(\zeta_{k}^{\prime}, v\left(\zeta_{k}^{\prime}\right)\right) .
$$

From (4.20) and (4.22) there exists a constant $K_{1}$ such that

$$
\begin{aligned}
& \left|H\left(\zeta_{k}, u\left(\zeta_{k}\right)\right)-H\left(\zeta_{k}^{\prime}, v\left(\zeta_{k}^{\prime}\right)\right)\right| \\
& \leq K_{1}\left[\frac{\left|\zeta_{k}\right|^{r+\frac{n-1}{n}}}{\left.|\log | \zeta_{k}\right|^{\frac{1}{n}}}\left|u\left(\zeta_{k}\right)-v\left(\zeta_{k}^{\prime}\right)\right|+\left|\zeta_{k}\right|^{r+1+\frac{n-1}{n}}|\log | \zeta_{k}||^{|J|}\left|\zeta_{k}-\zeta_{k}^{\prime}\right|\right] .
\end{aligned}
$$


On the other hand, using (4.35) we can write

$$
\left|v\left(\zeta_{k}^{\prime}\right)-u\left(\zeta_{k}\right)\right| \leq\left|\zeta_{k}\right||\log | \zeta_{k}||^{|J|}\left|\zeta_{k}^{\prime}-\zeta_{k}\right|+\left|\zeta_{k}\right|^{2}|\log | \zeta_{k}||^{|J|}\left\|h_{2}-h_{1}\right\|_{\infty},
$$

and by Lemma 4.16 it follows that

$$
\begin{aligned}
\left|v\left(\zeta_{k}^{\prime}\right)-u\left(\zeta_{k}\right)\right| \leq & \left(K\left|\zeta_{k}\right||\log | \zeta_{k}||^{|J|}|\zeta|^{3-\frac{1}{n}}|\log | \zeta||^{|J|-\frac{1}{n}}+\left.\left|\zeta_{k}\right|^{2}|\log | \zeta_{k}\right|^{|J|}\right) \\
& \cdot\left\|h_{2}-h_{1}\right\|_{\infty} .
\end{aligned}
$$

Applying Corollary 4.13, we get

$$
\left|\mathcal{R}_{1}\right| \leq\left. K_{7}|\zeta|^{2}|\log | \zeta\right|^{|J|-1}\left\|h_{2}-h_{1}\right\|_{\infty},
$$

for some constant $K_{7}$. In fact, there exist constants $K_{i}$, with $i=2, \ldots, 7$, such that

$$
\begin{aligned}
\left|\mathcal{R}_{1}\right| \leq & K_{1}|\zeta|^{\frac{1}{n}} \\
& \sum_{k=0}^{\infty} \frac{1}{\left|\zeta_{k}\right|^{\frac{1}{n}}}\left|\frac{\left|\zeta_{k}\right|^{r+\frac{n-1}{n}}}{\left.|\log | \zeta_{k}\right|^{\frac{1}{n}}}\right| u\left(\zeta_{k}\right)-\left.v\left(\zeta_{k}^{\prime}\right)|+| \zeta_{k}\right|^{r+1+\frac{n-1}{n}}|\log | \zeta_{k} \|^{|J|}\left|\zeta_{k}-\zeta_{k}^{\prime}\right| \mid \\
\leq & \left.K_{2}|\zeta|^{3}|\log | \zeta||^{|J|-\frac{1}{n}}\left\|h_{2}-h_{1}\right\|_{\infty} \sum_{k=0}^{\infty}\left|\zeta_{k}\right|^{r+1+\frac{n-2}{n}}|\log | \zeta_{k}\right|^{|J|-\frac{1}{n}} \\
& +K_{1}|\zeta|^{\frac{1}{n}}\left\|h_{2}-h_{1}\right\|_{\infty} \sum_{k=0}^{\infty}\left|\zeta_{k}\right|^{r+2+\frac{n-2}{n}}|\log | \zeta_{k}||^{|J|-\frac{1}{n}} \\
& +K_{3}|\zeta|^{3}|\log | \zeta||^{|J|-\frac{1}{n}}\left\|h_{2}-h_{1}\right\|_{\infty} \sum_{k=0}^{\infty}\left|\zeta_{k}\right|^{r+1+\frac{n-2}{n}}|\log | \zeta_{k} \|^{|J|} \\
\leq & \left.K_{4}|\zeta|^{4-\frac{1}{n}}|\log | \zeta\right|^{2|J|-1-\frac{1}{n}}\left\|h_{2}-h_{1}\right\|_{\infty}+\left.K_{5}|\zeta|^{2}|\log | \zeta\right|^{|J|-1}\left\|h_{2}-h_{1}\right\|_{\infty} \\
& +\left.K_{6}|\zeta|^{4-\frac{1}{n}}|\log | \zeta\right|^{2|J|-1}\left\|h_{2}-h_{1}\right\|_{\infty} \leq K_{7}|\zeta|^{2}|\log | \zeta||^{|J|-1}\left\|h_{2}-h_{1}\right\|_{\infty} .
\end{aligned}
$$

We can estimate the term $\mathcal{R}_{2}$ as follows: first we can write

$$
\left|\zeta_{k}^{-\frac{1}{n}}-\left(\zeta_{k}^{\prime}\right)^{-\frac{1}{n}}\right|=\left|\zeta_{k}^{-\frac{1}{n}}\right|\left|1-\exp \left(-\frac{1}{n} \log \frac{\zeta_{k}^{\prime}}{\zeta_{k}}\right)\right| ;
$$

then by (4.20) and since $v \in \mathcal{F}_{n}^{|J|}(\delta)$, it follows that

$$
\left|1-\exp \left(-\frac{1}{n} \log \frac{\zeta_{k}^{\prime}}{\zeta_{k}}\right)\right|\left|H\left(\zeta_{k}^{\prime}, v\left(\zeta_{k}^{\prime}\right)\right)\right| \leq K_{1}^{\prime} \frac{\left|\zeta_{k}^{\prime}-\zeta_{k}\right|}{\left|\zeta_{k}\right|}\left|\zeta_{k}\right|^{r+2+\frac{n-1}{n}}|\log | \zeta_{k}||^{|J|},
$$

and using Lemma 4.16, we get

$$
\begin{aligned}
& \left|1-\exp \left(-\frac{1}{n} \log \frac{\zeta_{k}^{\prime}}{\zeta_{k}}\right)\right|\left|H\left(\zeta_{k}^{\prime}, v\left(\zeta_{k}^{\prime}\right)\right)\right| \\
& \quad \leq\left.\left. K_{2}^{\prime}|\zeta|^{3-\frac{1}{n}}|\log | \zeta\right|^{|J|-\frac{1}{n}}\left\|h_{2}-h_{1}\right\|_{\infty}\left|\zeta_{k}\right|^{r+1+\frac{n-1}{n}}|\log | \zeta_{k}\right|^{|J|}
\end{aligned}
$$

where $K_{1}^{\prime}$ and $K_{2}^{\prime}$ are some constants. Finally by Corollary 4.13

$$
\left|\mathcal{R}_{2}\right| \leq\left. K_{3}^{\prime}|\zeta|^{4-\frac{1}{n}}|\log | \zeta\right|^{2|J|-1}\left\|h_{2}-h_{1}\right\|_{\infty},
$$

for some constant $K_{3}^{\prime}$. 
We observe that the term $\mathcal{R}_{2}$ is of smaller order than $\mathcal{R}_{1}$. Hence, for $\delta$ small enough, there exists a constant $K^{\prime}$ such that

$$
|T u(\zeta)-T v(\zeta)| \leq\left. K^{\prime}|\zeta|^{2}|\log | \zeta\right|^{|J|-1}\left\|h_{2}-h_{1}\right\|_{\infty} \leq\left. C|\zeta|^{2}|\log | \zeta\right|^{|J|} \mid h_{2}-h_{1} \|_{\infty},
$$

for a suitable $C<1$. By definition of the norm in $\mathcal{F}_{n}^{|J|}(\delta)$, this implies that, for $\delta$ small enough, we have

$$
\|T u-T v\| \leq C\|u-v\| .
$$

So $T$ is a contraction.

Since $n \geq 2$, we observe that $D_{r+\frac{n-1}{n}, \delta}$ has $r+1$ connected (and simply connected) components having $O$ on their boundary. Hence, if $\hat{f}$ is defined by (4.6) or (4.7), we obtain $r+1$ parabolic curves for $\hat{f}$ at the origin. These parabolic curves are distinct as sets. We think that the union of any two curves is not contained in a larger parabolic curve, but for now we do not have a proof of this statement. However, there exists at least one parabolic curve for $\hat{f}$ at the origin, and hence there exists at least one parabolic curve at the origin for $f$ given by (4.5).

This completes the proof of Theorem 4.6. and hence of our main result, Theorem 1.6 .

\section{ACKNOWLEDGEMENTS}

The author would like to thank M. Abate for his encouragement and support during the preparation of this article. She also thanks the referee for useful comments and suggestions.

\section{REFERENCES}

1. Abate, M., Diagonalization of nondiagonalizable discrete holomorphic dynamical systems. Amer. J. Math., 122 (2000), no. 4, 757-781. MR1771573 (2001m:32036)

2. Abate, M., The residual index and the dynamics of holomorphic maps tangent to the identity. Duke Math. J., 107 (2001), no. 1, 173-207. MR.1815255 (2003a:32028)

3. Abate, M., Bracci, F. \& Tovena, F., Index Theorems for holomorphic self-maps. Ann. of Math., 159 (2004), no. 2, 819-864. MR2081441 (2005g:32044)

4. Abate, M. \& Tovena, F., Parabolic curves in $\mathbb{C}^{3}$. Abstr. Appl. Anal., (2003), no. 5, 275-294. MR:1981266 (2004c:32035)

5. Camacho, C. \& Sad, P., Invariant varieties through singularities of holomorphic vector fields. Ann. of Math., (2) 115 (1982), no. 3, 579-595. MR657239 (83m:58062)

6. Carleson, L. \& Gamelin, T. W., Complex Dynamics. Springer-Verlag, New York, 1993. MR 1230383 (94h:30033)

7. Écalle, J., Les fonctions résurgentes, Tome III: L'équation du pont et la classification analytique des objects locaux. Publ. Math. Orsay, 85-5, Université de Paris-Sud, Orsay, 1985. MR $852210(87 \mathrm{k}: 32009)$

8. Fatou, P., Sur les équations fonctionnelles. Bull. Soc. Math. France, 47 (1919), 161-271. MR 1504787

9. Hakim, M., Analytic transformations of $\left(\mathbb{C}^{p}, 0\right)$ tangent to the identity. Duke Math. J., 92 (1998), no. 2, 403-428. MR.1612730(99a:32036)

10. Hakim, M., Stable pieces of manifolds in transformations tangent to the identity. Preprint, 1998. MR.1612730 (99a:32036)

11. Leau, L., Étude sur les équations fonctionelles à une ou plusieurs varibles. Ann. Fac. Sci. Toulouse, 11 (1897), E1-E110.

12. Ueda, T., Analytic transformations of two complex variables with parabolic fixed points. Preprint, 1997. 
13. Wasow, W., Asymptotic expansions for ordinary differential equations. Pure and Applied Mathematics, Vol. XIV Interscience Publishers John Wiley $\&$ Sons, Inc., New York-LondonSydney, 1965. MR0203188 (34:3041)

14. Weickert, B. J., Attracting basins for automorphisms of $\mathbb{C}^{2}$. Invent. Math., 132 (1998), no. 3, 581-605. MR1625716 (99e:32045)

Dipartimento di Matematica, Università degli Studi di Parma, Viale G. P. Usberti 53/A, I-43100, Parma, Italy

E-mail address: laura.molino@unipr.it 